TRANSACTIONS OF THE

AMERICAN MATHEMATICAL SOCIETY

Volume 364, Number 8, August 2012, Pages 3967-3992

S 0002-9947(2012)05424-9

Article electronically published on March 22, 2012

\title{
DIVISIBILITY THEORY IN COMMUTATIVE RINGS: BEZOUT MONOIDS
}

\author{
P. N. ÁNH, L. MÁRKI, AND P. VÁMOS \\ Dedicated to Bruno Bosbach
}

\begin{abstract}
A ubiquitous class of lattice ordered semigroups introduced by Bosbach in 1991, which we will call Bezout monoids, seems to be the appropriate structure for the study of divisibility in various classical rings like GCD domains (including UFD's), rings of low dimension (including semihereditary rings), as well as certain subdirect products of such rings and certain factors of such subdirect products. A Bezout monoid is a commutative monoid $S$ with 0 such that under the natural partial order (for $a, b \in S$, $a \leq b \in S \Longleftrightarrow b S \subseteq a S), S$ is a distributive lattice, multiplication is distributive over both meets and joins, and for any $x, y \in S$, if $d=x \wedge y$ and $d x_{1}=x$, then there is a $y_{1} \in S$ with $d y_{1}=y$ and $x_{1} \wedge y_{1}=1$. In the present paper, Bezout monoids are investigated by using filters and $m$-prime filters. We also prove analogues of the Pierce and the Grothendieck sheaf representations of rings for Bezout monoids. The question as to whether Bezout monoids describe divisibility in Bezout rings (rings whose finitely generated ideals are principal) is still open.
\end{abstract}

\section{INTRODUCTION}

Investigating divisibility in rings is as old as ring theory itself: the study of semigroups of divisibility of commutative rings (i.e., multiplicative semigroups of principal ideals partially ordered by reverse inclusion) originated from the factorization problems of algebraic integers. This factorization problem led Dedekind to introduce 'ideal numbers' and what we now call Dedekind rings. The next step was Krull's general valuation theory which established a dictionary between (the positive cones of) totally ordered abelian groups and (valuation domains of) valued fields. This was followed by the study of domains with varying degrees of 'nice' divisibility theories such as unique factorization domains or, more generally, greatest common divisor domains, as well as Dedekind and Prüfer domains. Krull's dictionary was extended several decades later by Jaffard 23] and Ohm 33] to lattice-ordered abelian groups and Bezout domains.

Received by the editors June 3, 2010.

2010 Mathematics Subject Classification. Primary 06F05; Secondary 13A05, 13F05, 20M14.

Key words and phrases. Bezout ring, divisibility semigroup, Bezout monoid, spectrum, prime filter, sheaf.

The first author was partially supported by the Hungarian National Foundation for Scientific Research grant no. K61007 and by the Colorado College and the University of Colorado at Colorado Springs during his stay at Colorado College in the fall of 2006.

The second author was partially supported by the Hungarian National Foundation for Scientific Research grant no. NK72523.

The third author acknowledges the hospitality of the Rényi Institute during the initial research phase of this paper. 
All these domains, and indeed some of their subdirect products and factors, share the property that certain ideals with respect to multiplication form a semigroup admitting a natural distributive lattice structure. It is important to note that the meet in this distributive lattice is, in general, not necessarily the sum of the ideals, e.g., the GCD of $x$ and $y$ in a polynomial ring $k[x, y]$ in two variables $x, y$ over a field $k$ is 1 , which generates the whole ring, and not the proper ideal generated by $x$ and $y$.

Now the question arises naturally: what is the class of rings and the appropriate abstract framework for studying divisibility in rings?

In terms of semigroups, the starting point is the celebrated Jaffard-Ohm Theorem (see [23, 24], 33]): the positive cone of every lattice-ordered abelian group arises as the semigroup of divisibility of a Bezout domain. Since the divisibility theory of any GCD domain or of any Prüfer domain is clearly the positive cone of a lattice-ordered abelian group, the divisibility theory of these much wider classes of rings reduces to the divisibility theory of Bezout domains, in which the divisibility relation is restricted to principal ideals.

Leaving the somewhat restrictive class of domains, we encounter Bezout rings (which may have zero-divisors) in Kaplansky's treatment of elementary divisor rings [27. We will focus on general Bezout rings in our considerations. There are two special cases of these which serve as examples we would like to generalize. The case of a valuation ring (not necessarily a domain) is reasonably straightforward in view of a result of Clifford [13 (see [35]) and likewise the other special case, von Neumann regular rings, whose semigroups of divisibility are obviously the Boolean algebras with product equal to join (see Proposition 1.16 below). In this paper we wish to lay the foundations for a satisfactory characterization/representation for the divisibility of Bezout rings, encompassing these two extreme cases. Indeed, there is evidence for our hope: the divisibility structure of semihereditary Bezout rings is exactly the semihereditary Bezout monoids (see below), where semihereditariness in the second case is defined with one of the equivalent definitions in the ring case: the set of annihilators of any element is the principal filter generated by some idempotent. This result is presented in a subsequent paper [3], its proof is based on the theory developed below.

Let us turn to the semigroup framework we are proposing. Divisibility in rings means the study of multiplication up to units or, more generally, the study of multiplication of (finitely generated) ideals. In the most general case, there seems to be no tool for describing this. There is a notion, however, which works in all the classical examples listed above: a class of lattice-ordered monoids introduced by Bosbach in 9] - without a proper name - which we will call here Bezout monoids. For the precise definition see Section 1 and ultimately Definition 1.5

For the purpose of an abstract description of divisibility in Bezout rings, in the present paper we initiate a careful study of Bezout monoids, with the aim of moving from the axiomatization through main properties to representation theory. Sheaf representation is a very powerful tool in commutative algebra, and we found that Bezout monoids are amenable to this treatment. In fact, we were able to find analogues of both the Pierce and the Grothendieck sheaf representations; see Section 4 and in particular Theorems 4.5 and4.10. We do not intend to present a full account of results on Bezout monoids. Clearly, results for more general classes like (distributive) lattice-ordered semigroups or $D$-monoids (Bosbach's $d$-semigroups or 
divisibility semigroups) are valid for Bezout monoids. Here we present only results which we think to be useful towards an abstract characterization of the divisibility structure of Bezout rings. Among the results presented here, there are some, mostly elementary in character, which can be found sometimes hidden and scattered in the literature, many of them in Bosbach's papers. For a related topic see Bosbach [11. We were surprised to find how ubiquitous Bezout monoids are; see some of the examples in Section 1 .

The topic of our paper belongs to the wider field of Multiplicative Ideal Theory, which is treated in the - by now - classical books of Larsen and McCarthy [30] and Gilmer [19]. Note that the theory of arithmetical rings introduced by Fuchs [16] also belongs to Multiplicative Ideal Theory: these rings arose as Fuchs generalized the classical Lasker-Noether theory to the non-noetherian case. Within Multiplicative Ideal Theory, axiomatic approaches without making reference to the additive structure started in 1939. One branch of this direction, the study of multiplicative lattices, goes back to the paper of Ward and Dilworth [40; two major papers in this line are those of Dilworth [14 and Anderson 11. Another branch, that of ideal systems, goes back to Lorenzen [31] and was continued by Jaffard 24] and Aubert [4] among others. For a fairly up-to-date presentation see Halter-Koch [21]. Bosbach's theory, on which our approach is based, also goes back to the line started by Lorenzen. In spite of many connections with other directions, Bosbach's work has not yet been integrated into a general picture of Abstract Multiplicative Ideal Theory.

A word about terminology. For several notions, we decided to use names different from the ones introduced earlier (some of these notions had different names by different authors). The names we use refer both to the order and the multiplicative aspect, and they are intended to emphasize that the goal of this theory is an abstract description of the divisibility structure of (Bezout) rings. In the earlier terminologies, this background was not always clearly visible.

The authors are grateful to Bruno Bosbach and Klaus Keimel for many helpful hints about earlier related work in this topic, as well as to those colleagues whose criticisms on an earlier version of this paper was a great help in improving the presentation.

\section{BASIC DEFINITIONS AND PRELIMINARY RESULTS}

\section{Notation.}

- The extension of a semigroup $S$ with an additional zero element is denoted by $S^{\bullet}$.

- For subsets $U, V$ of a semigroup or a ring, define the quotient $U: V=$ $\{x \mid x V \subseteq U\}$ and write $u: V, U: v$, or $u: v$ in the case of $U$ or $V$ having just one element $u$ or $v$, respectively.

\section{Terminology.}

- All structures are commutative, and rings have an identity element 1.

- A monoid is a semigroup with identity.

- A monoid with zero is called cancellative if its nonzero elements form a cancellative monoid and 0 -cancellative if $x z=y z \neq 0$ implies $x=y$ for all $x, y, z$. 
- The natural quasi-order on a monoid $S$ is the relation defined by divisibility: $a \leq b \Longleftrightarrow a S \supseteq b S \Longleftrightarrow a \mid b$. If this quasi-order is a partial order, then we speak of natural partial order and call the monoid a naturally partially ordered monoid. In such a monoid, 1 is the unique invertible element, or equivalently, 1 is multiplicatively irreducible (i.e., $a b=1$ iff $a=1=b$ for all $a, b \in S$ ).

- An element $s$ of a partially ordered monoid $S$ is said to be positive if $s \geq 1$. The set $S^{+}$of positive elements is a submonoid called the positive cone of $S$. Clearly, every element is positive in a naturally partially ordered monoid.

- An $l$-monoid is a lattice-ordered monoid $S$ in which multiplication distributes over the lattice operations: $c(a \wedge b)=c a \wedge c b$ and $c(a \vee b)=c a \vee c b$ for all $a, b, c \in S$.

- A distributive l-monoid is an l-monoid whose underlying lattice is distributive.

- A $D$-monoid is a distributive $l$-monoid with a 0 element whose order is the natural partial order.

It is obvious that the positive cone of an abelian lattice-ordered group together with a zero element is a $D$-monoid. However, in contrast to lattice-ordered groups, if we consider a lattice-ordered monoid with zero whose underlying lattice is distributive and in which multiplication distributes over the lattice operations, then its positive elements need not form a $D$-monoid. In other words, the induced order on positive elements is not necessarily the natural one, as was shown in [5, Chapter XIV.4]. In order for this induced order to be the natural one, a mild but decisive condition is needed (see the following proposition), which characterizes $D$ monoids among $m$-semilattices (see [5, Chapter XIV.4]); this latter structure was first studied in [15].

Proposition 1.1. If $S$ is a distributive l-monoid, then $S^{+}$is a $D$-monoid if and only if, for all $a, b \in S^{+}, a \wedge b=a$ implies $b=a x$ for some $x \in S$.

Proof. All we have to prove is that this element $x$ can be chosen from $S^{+}$. Indeed, if both $a, b \geq 1$ and $a \wedge b=a$, and $b=a x$ for some $x$, then $c=x \vee 1 \geq 1$ and $a c=a(x \vee 1)=a x \vee a=b \vee a=b$.

Note that our definition of $D$-monoids is redundant and is given in this form for the sake of better readability. In fact, we have:

Proposition 1.2 (Bosbach [7, 2.7-2.11]). If a monoid $S$ with 0 is a meet semilattice under the natural order such that products are distributive over meets, then $S$ is a distributive lattice, multiplication also distributes over joins, and $a \wedge b=1$ implies $a \vee b=a b$.

For the reader's sake we include the proof.

Proof. Given any two elements $a, b \in S$, put $d=a \wedge b, a=d a_{1}, b=d b_{1}$. We claim that the element $a b_{1}=d a_{1} b_{1}=a_{1} b$ is the least upper bound $a \vee b$ of $a$ and $b$. For, if $c \geq a, b$, then write $c=a c_{1}$, and $b=b \wedge c \wedge b c_{1}=b \wedge a c_{1} \wedge b c_{1}=d b_{1} \wedge(a \wedge b) c_{1}=$ $d\left(b_{1} \wedge c_{1}\right)$ implies $b a_{1}=d a_{1}\left(b_{1} \wedge c_{1}\right)=a\left(b_{1} \wedge c_{1}\right) \leq a c_{1}=c$. Thus $S$ is a lattice, and it is clear that multiplication distributes over this join. To show that $S$ is a distributive lattice, it is enough to check the inequality $y=(a \vee b) \wedge(a \vee c) \leq$ $a \vee(b \wedge c)=x$ for arbitrary $a, b, c \in S$. Indeed, if $d=a \wedge b \wedge c, a=d a_{1}$ for some 
$a_{1} \in S$, then $x=a_{1}(b \wedge c)=a_{1} b \wedge a_{1} c \geq y$ holds in view of $a \vee b \leq a_{1} b, a \vee c \leq a_{1} c$. From the above presentation of the join it follows immediately that $a \wedge b=1$ implies $a \vee b=a b$.

The implication $a \wedge b=1 \Longrightarrow a \vee b=a b$ will often be used in the sequel, without mentioning it explicitly.

There are several classes of semigroups modelled on divisibility in a domain. One of these is the divisibility monoids introduced in Birkhoff's book [5]. This is a monoid equipped with the natural partial order. However, divisibility monoids in this sense cannot, in general, be realized as monoids of principal ideals of a commutative ring even if the order is total. Indeed, if the principal ideals of a commutative ring form a chain, then their monoid is 0 -cancellative, but a naturally totally ordered monoid with 0 need not be 0-cancellative (see Clifford [13]). A more satisfactory generalization of the positive cone of a lattice-ordered group was introduced by Bosbach [7. The notion we are going to use, namely Bezout monoid, or simply B-monoid, evolved over the decades in his works and culminated in the positive hyper-normal commutative divisibility semigroups with 0 and 1 (see in [9, Section 4]).

Definition 1.3. A monoid $S$ is said to be hyper-normal if for any $a=a e \in S$ and $b \in a S$ there is $z \in S$ such that $b=a z$ and $S$ is the unique principal ideal containing both $e$ and $z$.

In a $D$-monoid $S$, hyper-normality has a formally stronger but equivalent form. (In fact, this is how Bosbach defined hyper-normality, and he also showed the equivalence of the two conditions in a $D$-monoid in [9, Lemma 4.2].) First of all notice that, in a $D$-monoid, the fact that $S$ is the unique principal ideal containing both $e$ and $z$ is simply expressed by $e \wedge z=1$.

Proposition 1.4 (cf. Bosbach [9, Lemma 4.2]). A D-monoid $S$ is hyper-normal if and only if, for any $x, y \in S$, if $d=x \wedge y$ and $d x_{1}=x$, then there is a $y_{1} \in S$ with $d y_{1}=y$ and $x_{1} \wedge y_{1}=1$.

Proof. Definition 1.3 is the special case of the condition in our statement for $d=x$, as seen by putting $a=d=x, b=y, e=x_{1}, z=y_{1}$. Conversely, if a $D$-monoid $S$ is hyper-normal in the sense of Definition 1.3 and $d=x \wedge y=d\left(x_{1} \wedge y_{2}\right) \leq$ $b=d y_{2}, x=d x_{1}$, then one has $b=d z$ with $1=\left(x_{1} \wedge y_{2}\right) \wedge z=x_{1} \wedge\left(y_{2} \wedge z\right)$ and $b=d y_{2}=d z=d\left(y_{2} \wedge z\right)$, which implies hyper-normality in the above stronger form with $y_{1}=y_{2} \wedge z$.

This stronger form of hyper-normality corresponds formally to an elementary result in number theory: one can take out the greatest common divisor to obtain coprime natural numbers. However, one has to be very careful in dealing with this idea. A Bezout ring satisfying hyper-normality for elements instead of principal ideals is called Hermitian. But not every Bezout ring is Hermitian; see e.g. [18, Example 3.4].

Notice that the semigroup of divisibility of an arbitrary ring is hyper-normal even if the principal ideals do not form a lattice, in which case they do not form a $D$-monoid. For, if $R$ is an arbitrary ring, then $a R \cdot e R=a R$ implies $a=a e c$ for some $c \in R$, and if $a R \leq b R$, that is, $b \in a R$, then $b=a x$ for some $x \in R$. Hence $a x=a(e c+e c x-1)$, and therefore $a R \supseteq b R=a x R=a R \cdot[e(c+c x)-1] R$ and 
$[e(c+c x)-1] R+e R=R$. We do not know if the semigroup of finitely generated ideals of an arbitrary commutative ring is hyper-normal.

Definition 1.5. A Bezout monoid, or B-monoid for short, is a hyper-normal $D$ monoid.

It is obvious that the positive cone of an abelian lattice-ordered group together with a zero element is a $B$-monoid. Another source of examples is arithmetical rings, a notion introduced by Fuchs [16].

Recall that a ring is said to be arithmetical if its ideals form a distributive lattice. Arithmetical rings are, among others, the subject of a series of exercises in the books of Gilmer [19] (25.19) and Larsen and McCarthy 30. (IV.18). In fact, most of these results go back to Jensen. In particular, Theorem 2 of Jensen 26] says that a ring is arithmetical if and only if, for any pair of ideals $A, B$ such that $A \subseteq B$ and $B$ is finitely generated, there exists an ideal $C$ for which $A=B C$. Since every ideal is the union of the finitely generated ideals contained in it, this characterization remains valid if $A$ and $C$ are also required to be finitely generated, and thus an equivalent version of Jensen's theorem states that a ring is arithmetical if and only if the partial order in the monoid of finitely generated ideals ordered by reverse inclusion is the natural one. As an easy consequence of this result, one can obtain examples of $B$-monoids using the following assertion, which can be seen immediately from results of Anderson [2] and Bosbach [9]. However, for the reader's sake we include a direct proof based on Jensen's theorem.

Proposition 1.6. The semigroup $S$ of finitely generated ideals in a ring $R$ is a $B$-monoid if and only if $R$ is an arithmetical ring. In particular, the semigroup of divisibility of a Bezout ring is a B-monoid.

Proof. Let $S$ be the monoid of finitely generated ideals in a ring $R$. If $S$ is a $B$-monoid, then $R$ is arithmetical by Jensen's theorem. Conversely, if $R$ is arithmetical, then, under the partial order defined by reverse inclusion, $A \wedge B=A+B$ for any $A, B \in S$. Hence $S$ is a meet semilattice, and $C(A \wedge B)=(C A \wedge C B)$ for all $A, B, C \in S$. By Proposition 1.2 it follows that $S$ is a distributive lattice, where the join also commutes with multiplication. To prove that it is a $B$-monoid, we have to verify that $S$ is hyper-normal. Let $I, J, K$ be finitely generated ideals with $K \subseteq I J=I$. Again by Jensen's theorem the equality $K=I(K: I)$ holds. Consequently, by Proposition 1.4 it suffices to show that $R=J+(K: I)$. For any maximal ideal $M$ of $R$, the Nakayama Lemma implies $J_{M}=R_{M}$ in the case $I_{M} \neq 0$ in view of the equality $I_{M}=(I J)_{M}=I_{M} J_{M}$. Therefore the equality $[J+(K: I)]_{M}=J_{M}+\left(K_{M}: I_{M}\right)=R_{M}$ holds because by using [26, Lemma on p. 116] we have $(K: I)_{M}=\left(K_{M}: I_{M}\right)$, hence $R=J+(K: I)$.

The following statement might be known to experts in commutative algebra, although we could not find any explicit reference. The statement is known for Bezout rings (see Larsen, Lewis and Shores [29]), and then the more general statement for arithmetical rings follows by Anderson [2, Corollary 8.1]. For Prüfer domains and regular rings, which are semihereditary and hence coherent, it is an obvious consequence of the characterization of coherent rings in [37, Proposition I.13.3]. Here we give a simple direct proof. Note that the procedure described in the proof of Proposition 1.2 for getting the join of two elements in a $B$-monoid also gives a way to find generators for the intersection of two finitely generated ideals in an arithmetical ring. 
Proposition 1.7. The intersection of two finitely generated ideals in an arithmetical ring is again finitely generated.

Proof. Let $R$ be an arithmetical ring, and consider the lattice of its finitely generated ideals. Since the natural order coincides with reverse inclusion, it follows that, for any two finitely generated ideals $A, B$ of $R, A \vee B$ is the greatest finitely generated ideal contained in $A \cap B$. However, this implies $A \vee B=A \cap B$ since $A \cap B$ is the union of its finitely generated ideals.

We continue with examples for $B$-monoids.

\section{Examples 1.8.}

(1) The best known examples for $B$-monoids are semigroups of divisibility of Bezout rings; see Proposition 1.6. Examples for Bezout rings include valuation rings as well as Bezout domains and their factor rings. Notice, however, that not all Bezout rings can be obtained as factor rings of Bezout domains - this fails even for valuation rings, as shown by the example constructed by Fuchs and Salce [17. To get more sophisticated examples of Bezout rings we can use a pull-back construction. To this end let $V$ be a valuation ring (not necessarily a domain) with residue field $K$, and take a Bezout subring $C$ of $K$ whose field of quotients is $K$. Then the inverse image of $C$ under the canonical surjection $V \rightarrow K$ is a Bezout ring, and so is any of its factor rings. In this way one can obtain Bezout rings with interesting properties, e.g., Bezout rings with a minimal prime ideal which is a direct sum of (possibly infinitely many) valuation modules (see [36]) or elementary divisor domains which are not adequate domains (cf. [22, Example 1]).

Bezout rings and their proper subclasses like those of Hermitian rings or of elementary divisor rings, etc., appear naturally in Kaplansky's treatment of diagonalization of not necessarily square matrices and, in particular, of elementary divisor rings. He left it open if they are in fact proper subclasses of Bezout rings. Gillman and Henriksen [18] defined subclasses of completely regular Hausdorff spaces $X$ by requiring that the corresponding ring $C(X)$ of all continuous real-valued functions defined on $X$ be Bezout, Hermitian, von Neumann regular, or elementary divisor rings. In this way they obtained the notions of $F-, T$-, $P$ - and $D$-spaces, respectively, and showed that these form pairwise different classes of topological spaces, thereby answering positively the algebraic questions which have arisen in the above-mentioned theory of Kaplansky. For more information and further examples about Bezout rings, see e.g. [18, 27, [29].

(2) In the special case of a Prüfer domain (in particular, in a Dedekind domain) the $B$-monoid of finitely generated ideals is cancellative.

(3) Semigroups of divisibility of unique factorization domains or, more generally, of greatest common divisor domains are also $B$-monoids with GCD and LCM as $\wedge$ and $\vee$, respectively. Such is, in particular, every polynomial ring in an arbitrary number of indeterminates over a field. Thus, although in all the examples listed above the join operation is given by the intersection of the corresponding ideals, meets need not be the generated ideals, as we have seen in the introduction. 
(4) Several classes of $D$-monoids have been defined by means of their arithmetics (calculation rules) and extensively investigated mainly for their decomposition and extension properties by Bosbach. Among these, complementary semigroups [6] and bricks [8] are $B$-monoids. (Moreover, every brick is isomorphic to the divisibility structure of some Bezout ring [8.)

Remarks 1.9.

(1) In view of Proposition [1.6 it is natural to ask if every $B$-monoid can be obtained as the semigroup of finitely generated ideals of an arithmetical ring partially ordered by reverse inclusion. By Anderson [2, Corollary 8.1], this is equivalent to the seemingly stronger question as to whether every $B$-monoid can be realized as the semigroup of divisibility of an appropriate Bezout ring.

(2) The semigroup of finitely generated ideals in certain coherent rings, e.g., in non-Dedekind noetherian domains, shows that the order induced by reverse inclusion in the semigroup of finitely generated ideals is, in general, not the natural partial order in view of [26 Theorem 2. In addition, in the semigroup of ideals of a non-Dedekind noetherian domain, multiplication is distributive over meets (i.e., over sums of ideals) but not necessarily over joins, i.e., over set-theoretic intersections of ideals; this lattice-ordered semigroup is neither distributive nor cancellative. In fact, by Jensen 25, Lemma 2], a domain is a Prüfer domain iff ideal multiplication is distributive over set-theoretic intersections (i.e., joins) of ideals. Moreover, there are non-arithmetical rings (necessarily with non-zero nilpotent elements) such that ideal multiplication is distributive over intersections of ideals. See also [30, Theorem 10.18].

The following simple properties of $B$-monoids will be extremely important for our investigations. The first of them is obtained by easy induction based on hypernormality, and we omit its proof.

Proposition 1.10. Let $S$ be a $B$-monoid and let $s=s_{1} \wedge \cdots \wedge s_{n}$ in $S, n \geq 2$. Then there exist elements $t_{1}, \ldots, t_{n} \in S$ such that $t_{1} \wedge \cdots \wedge t_{n}=1$ and, for $i=1, \ldots, n$, $s_{i}=s t_{i}$.

Lemma 1.11. In a B-monoid $S, \bigwedge_{i=1}^{n} a_{i}=1$ implies $\bigwedge_{i=1}^{n} a_{i}^{m_{i}}=1$ for arbitrary $m_{i} \in \mathbb{N}$. In particular, for any $a, b$ it holds that $(a \wedge b)^{n}=a^{n} \wedge b^{n},(a \vee b)^{n}=$ $a^{n} \vee b^{n}$. Likewise, given arbitrary $a_{1}, \ldots, a_{n}, b_{1}, \ldots, b_{m}$, if $a_{i} \wedge b_{j}=1$ for all $i, j$, then $a_{1} \cdots a_{n} \wedge b_{1} \cdots b_{m}=1$.

Proof. If $m$ is the maximum of the $m_{i}$, then

$$
\bigwedge_{i=1}^{n} a_{i}^{m_{i}} \leq \bigwedge_{i=1}^{n} a_{i}^{m} \leq\left(\bigwedge_{i=1}^{n} a_{i}\right)^{n(m-1)+1}=1
$$

whence $\bigwedge_{i=1}^{n} a_{i}^{m_{i}}=1$ follows. Next, for arbitrary $a, b$ let $d=a \wedge b, a=d a_{1}, b=$ $d b_{1}, a_{1} \wedge b_{1}=1$ for appropriate $a_{1}, b_{1} \in S$. By the foregoing we have $a^{n} \wedge b^{n}=d^{n} a_{1}^{n} \wedge$ $d^{n} b_{1}^{n}=d^{n}\left(a_{1}^{n} \wedge b_{1}^{n}\right)=d^{n}=(a \wedge b)^{n}$ and $(a \vee b)^{n}=d^{n} a_{1}^{n} b_{1}^{n}=d^{n}\left(a_{1}^{n} \vee b_{1}^{n}\right)=a^{n} \vee b^{n}$. The proof of the last claim is similar.

Proposition 1.12. If 1 is a meet-irreducible element of a $B$-monoid $S$, i.e., $s \wedge t=$ 1 implies $s=1$ or $t=1$, then $S$ is a 0 -cancellative chain. 
Proof. Take arbitrary elements $s_{1}, s_{2} \in S$ and put $s=s_{1} \wedge s_{2}$. Then there exist $t_{1}, t_{2} \in S$ such that $s_{1}=s t_{1}$ and $s_{2}=s t_{2}$ with $t_{1} \wedge t_{2}=1$. Hence $s=s_{1}$ or $s=s_{2}$, that is, $s_{1}$ and $s_{2}$ are comparable. Thus $S$ is a chain.

Let $a x=a y \neq 0$. Put $d=x \wedge y \leq x \vee y=d b$ and $c=a d$. The equality $a d=a(x \wedge y)=a x \wedge a y=a x \vee a y=a(x \vee y)=a d b \neq 0$ implies $b c=c \neq 0$. By hyper-normality there is an $e$ with $b \wedge e=1$ and $c e=0$. Thus $e \neq 1$ and so $b=1$, i.e., $x=y$, because 1 is meet-irreducible.

Remark 1.13. Notice the importance of hyper-normality here: the assertion does not hold for $D$-monoids in place of $B$-monoids. For example, an infinite set and its finite subsets form a $D$-monoid under set-theoretic intersection as multiplication and set-theoretic union as meet, which is not a chain although 1 is obviously meetirreducible. Hence the class of $B$-monoids does not form a variety, being not closed under subalgebras.

The following simple assertion underlines the importance of hyper-normality.

Proposition 1.14. If $S$ is a B-monoid, then the set $B(S)$ of all idempotents in $S$ is a Boolean algebra.

Proof. For $e, f \in B(S)$ one easily checks that $e \wedge f, e \vee f \in B(S)$, i.e, $B(S)$ is a (distributive) sublattice of $S$. For each $e \in B(S)$, the equality $e=e^{2} \leq 0$ implies by hyper-normality the existence of an element $f \in S$ with ef $=0, e \wedge f=1$. Therefore $f^{2}=f \in B(S)$ and $e \vee f=0$. Thus $f$ is the complement of $e$ and $B(S)$ is indeed a Boolean algebra.

Remark 1.15. In view of this result one can speak about the orthogonal complement of an idempotent in a $B$-monoid.

Proposition 1.16. Let $e, f$ be idempotents in a $B$-monoid $S$. Then $e \vee f=e f$.

Proof. Let $e, f \leq g \in S$. The natural order implies $g e=g=g f$. Hence ef $\leq$ gef $=g$, which completes the proof.

\section{Filters, $m$-PRIME Filters AND homomorPhisms}

In this section we develop the analogues of the ring concepts of ideal, prime ideal, radical and localization for $B$-monoids, culminating in the description of congruences and homomorphisms of $B$-monoids. The notion of ideals and prime ideals in semigroup theory will not be subtle enough for this purpose in $B$-monoids. For example, the semigroup $\mathbb{N}$ of non-negative integers under multiplication has uncountably many ideals, but as a $B$-monoid, i.e., endowed with the natural partial order, it has only countably many filters in the lattice theoretical sense - filters correspond to the elements of $\mathbb{N}$. This observation shows that filters may play a more natural role in the theory of $B$-monoids than semigroup ideals or semigroup prime ideals; it is filters which may complete the similarity between $B$-monoids and the multiplicative theory (i.e., the division relation) of Bezout rings. Indeed, finitely generated filters are principal in $B$-monoids.

In what follows, $S$ will always stand for a $B$-monoid. Recall that a filter is a subset $F \subseteq S$ closed under meets and 'greater': if $a, b \in F$, then $a \wedge b \in F$, and for $c \in F$, if $a \leq c$, then $c \in F$ also. Thus filters are also semigroup ideals of $S$. Clearly, if $F$ is a filter and $x \in S$, then $x F$ and $F: x$ are filters. Also recall that a filter $F$ is $\vee$-irreducible if $a \vee b \in F$ implies $a \in F$ or $b \in F$. For our purposes 
we need a stronger combination of the lattice and the multiplicative structures: we unify the notion of semigroup prime ideals and $\vee$-irreducible filters as follows.

Definition 2.1. A filter $F$ of $S$ is said to be $m$-prime if $F \neq S$ and if $a b \in F$ implies $a \in F$ or $b \in F$. Since $a \vee b \leq a b$ always holds in a $B$-monoid, an $m$-prime filter is necessarily $\vee$-irreducible.

Note that in most textbooks on lattice theory $\vee$-irreducible filters are called prime filters or sometimes dual prime ideals. However, these names would be less appropriate for us because we shall need the multiplicative prime property. For instance, all filters in a chain are $\vee$-irreducible, but in a totally ordered $B$-monoid not all $\vee$-irreducible filters are prime ideals under multiplication (i.e., $m$-prime filters).

If $X \subseteq S$, then the set

$$
X_{f}=\left\{x \in S \mid \text { there exist } r \in \mathbb{N} \text { and } x_{1}, \ldots, x_{r} \in X \text { such that } x \geq x_{1} \wedge \cdots \wedge x_{r}\right\}
$$

is the smallest filter containing $X$, and the map $f=\left(X \mapsto X_{f}\right)$ is an ideal system (Aubert $x$-system) on $S$ (as dealt with in Part A of Halter-Koch's book [21]). The $m$-prime filters are just the prime $f$-ideals. Several results proved in the sequel (in particular those concerning $m$-prime filters) are in fact consequences of the general theory of ideal systems. However, in order to be self-contained, we include their easy proofs.

For arbitrary subsets $U, V \subseteq S$, set

$$
\begin{gathered}
U \wedge V=\{u \wedge v \mid u \in U, v \in V\}, U \vee V=\{u \vee v \mid u \in U, v \in V\}, \\
U^{\perp}=\{s \in S \mid s U=0\},
\end{gathered}
$$

hence $U \wedge V, U \vee V$ are filters if $U, V$ are filters. In particular, $U^{\perp}(=0: U)$ is a filter for any subset $U$. For the sake of simplicity we write $u \wedge V, u \vee V$ and $u^{\perp}$ in the case $U$ is a subset with just one element $u$. We shall need some easy folklore results, which we nevertheless prove for the reader's sake. Krull's separation trick (well known for both commutative rings and distributive lattices) gives immediately:

Proposition 2.2. Let $P$ be a subset of $S, F_{0}$ a filter such that $F_{0} \cap P=\varnothing$ and $\Omega$ the set of all filters $F$ such that $F_{0} \subseteq F$ and $F \cap P=\varnothing$. Then $\Omega$ possesses maximal elements (with respect to inclusion), and if $F$ is such a maximal element, then:

(1) If $P$ is closed under $\vee$, then $F$ is $\vee$-irreducible.

(2) If $P$ is multiplicatively closed, then $F$ is m-prime. In particular, every maximal filter is m-prime.

Proof. Clearly, $\Omega$ has maximal elements by the Zorn Lemma. Notice that, for a filter $F$ and an element $a \in S$, the smallest filter containing $F$ and $a$ is $a S \wedge F$.

(1) Assume that $P$ is closed under $\vee$ and $x \vee y \in F, x, y \notin F$. Maximality of $F$ implies that there are $a, b \in F$ and $s, t \in S$ such that $x s \wedge a, y t \wedge b \in P$. Consequently,

$$
\begin{aligned}
P \ni(x s \wedge a) \vee(y t \wedge b) & =(x s \vee y t) \wedge(x s \vee b) \wedge(a \vee y t) \wedge(a \vee b) \\
& \geq(x \vee y) \wedge(x s \vee b) \wedge(a \vee y t) \wedge(a \vee b) \in F,
\end{aligned}
$$

a contradiction.

(2) Assume that $P$ is multiplicatively closed, $a b \in F$ and both $a S \wedge F, b S \wedge F$ contain $F$ properly. Then there are elements $x, y \in S$ and $u, v \in F$ such that $a x \wedge u, b y \wedge v \in P$, and hence we have $(a x \wedge u)(b y \wedge v) \in F \cap P$, a contradiction. 
As in the case of rings one can define the Jacobson radical of $S$ as the (set-theoreical) intersection of all maximal filters and the nil radical as the (set-theoretical) intersection of all $m$-prime filters. We call a $B$-monoid semiprime or reduced if its nil radical is 0 . More generally, the radical $I(F)$ of a filter $F$ is defined as the intersection of all $m$-prime filters containing $F$. In view of Proposition 2.2 we obtain the following result in the same way as for rings.

Corollary 2.3. For any filter $F$ of a B-monoid, its radical $I(F)$ consists of all elements $s \in S$ with $s^{n} \in F$ for some $n \in \mathbb{N}$. In particular, the nil radical is just the set of all nilpotent elements.

Definition 2.4. If the radical $I(F)$ of a filter $F$ is an $m$-prime filter $P$, then $F$ is called a primary filter, or more precisely, a $P$-primary filter. If 0 is a primary filter, then $S$ is called a primary $B$-monoid. Clearly, $S$ is a primary $B$-monoid iff $I(0)$ is the smallest $m$-prime filter of $S$.

Proposition 2.5. If $F$ is a $\vee$-irreducible filter in a $B$-monoid $S$, then $I(F)$ is the smallest $m$-prime filter containing $F$.

Proof. By Corollary $2.3 a b \in I(F)$ means $(a b)^{n} \in F$ for some $n \in \mathbb{N}$. Therefore $F \ni(a b)^{n} \leq(a \vee b)^{2 n}=a^{2 n} \vee b^{2 n} \in F$, and hence either $a^{2 n} \in F$ or $b^{2 n} \in F$, i.e., either $a \in \bar{I}(F)$ or $b \in I(F)$. Thus $I(F)$ is an $m$-prime filter. The minimality of $I(F)$ is clear.

Proposition 2.6. If a multiplicative prime ideal $I$ of $S$ is not a filter, then every element of $S$ is a meet of (two) elements from $I$.

Proof. If $I$ is not a filter, then it is not closed under $\wedge$, and hence there exist $c_{1}, c_{2} \in I$ such that $c_{1} \wedge c_{2}=c \notin I$. Now, by hyper-normality, there exist elements $d_{1}, d_{2} \in S$ with $d_{1} \wedge d_{2}=1$ and $c_{i}=c d_{i}, i=1,2$. By our assumptions, $d_{1}$ and $d_{2}$ and hence all their multiples must belong to $I$, and for every element $x \in S$ we have $x=x d_{1} \wedge x d_{2}$ with $x d_{1}, x d_{2} \in I$.

Remark 2.7. The condition $a b=1 \Longrightarrow a=1=b$ implies that $S \backslash 1$ is always a prime ideal, but it is usually not closed under meets. The most obvious example is the $B$-monoid of non-negative integers under multiplication.

Here we collect some basic, interesting results on $B$-monoids which are known for finitely generated ideals of arithmetical rings (cf. [37, [12]).

Proposition 2.8. For arbitrary $a, b \in S$ one has

$$
(S a: b) \wedge(S b: a)=S .
$$

Proof. Put $d=a \wedge b=d\left(a_{1} \wedge b_{1}\right), a=d a_{1}, b=d b_{1}, a_{1} \wedge b_{1}=1$ by using hypernormality. Then $d a_{1} b_{1}=a b_{1}=b a_{1}$, and hence $a_{1} \in(S a: b), b_{1} \in(S b: a)$ satisfying $a_{1} \wedge b_{1}=1$, and the assertion follows.

Remark 2.9. This result is not true for $D$-monoids: consider the example defined in Remark 1.13. Due to the lack of hyper-normality, several 'nice' properties of arithmetical rings do not carry over to $D$-monoids, in contrast to $B$-monoids.

Corollary 2.10. If $P$ and $Q$ are $m$-prime filters of $S$, then either $P \subseteq Q$ or $Q \subseteq P$ or $P \wedge Q=S$. In particular, every $m$-prime filter contains a unique minimal $m$ prime filter. If $P$ is an m-prime filter contained in the Jacobson radical, then 
for every element $a \in S$, either $a \in P$ or $P \subseteq S a$. Moreover, in this case $S$ is indecomposable, i.e., 1 and 0 are the only idempotents in $S$, and the nil radical of $S$ is the smallest $m$-prime filter of $S$.

Proof. Suppose that $P \nsubseteq Q$ and $Q \nsubseteq P$. Then there exist $p \in P \backslash Q, q \in Q \backslash P$. This implies $(S p: q) \subseteq P,(S q: p) \subseteq Q$, and hence $P \wedge Q=S$.

Suppose that an $m$-prime filter $P$ is contained in the Jacobson radical of $S$ and $a \notin P$. Then for any $p \in P$ we have the inclusion $(S p: a) \subseteq P$, and hence $P \wedge(S a: p) \supseteq(S p: a) \wedge(S a: p)=S$, which implies $(S a: p)=S$, i.e., $p \in S a$. Therefore in this case the prime ideals contained in the Jacobson radical form a chain, from which the other statements follow.

Proposition 2.2 provides ways to construct $m$-prime filters. The following result emphasizes the importance of $m$-prime filters. We call a $B$-monoid $S$ local if it has a unique maximal filter (maximal filters are understood to be different from $S$ ), or, equivalently, $S \backslash 1$ is a filter, or again, $x \wedge y=1$ if and only if $x=1$ or $y=1$. In view of Proposition 1.12, local $B$-monoids are 0-cancellative chains. Homomorphisms or congruences of $B$-monoids are meant in the sense of lattice-ordered semigroups or, equivalently (in view of Proposition 1.2), of multiplicative meet semilattices. Recall that, for a semigroup $S$ and an ideal $I$ of $S$, the Rees factor of $S$ by $I$ is the factor semigroup of $S$ by the congruence whose only non-singleton class is $I$.

Theorem 2.11. Let $T$ be a local B-monoid and $\varphi: S \longrightarrow T$ be a surjective homomorphism from a B-monoid $S$ onto $T$. Put $F=\{x \in S \mid \varphi(x) \neq 1\}$, $Q=\{x \in S \mid \varphi(x)=0\}$, and $K=\{x \in S \mid \exists s \notin F: s x=0\}$. Then $F \supseteq Q \supseteq K$ are filters and $F$ is $m$-prime. Moreover, we have for all $x, y \in S$ :

$$
\varphi(x)=\varphi(y) \neq 0 \text { if and only if }
$$

$x, y \notin Q$ and there is an $s \notin F$ with $x \leq y s$ and $y \leq x s$.

Conversely, for every $m$-prime filter $F$ the relation $\cong$ defined between the elements $x, y \in S$ by

$$
x \cong y \Longleftrightarrow \exists s \notin F: x \leq y s \text { and } y \leq x s
$$

is a congruence relation whose corresponding factor semigroup $S_{F}$ is a totally ordered B-monoid, and the congruence class of 0 is $K=\{x \in S \mid \exists s \notin F: s x=0\}$. In particular, if $\varphi: S \longrightarrow T$ is as above, then $T$ is the Rees factor of $S_{F}$ by the image $Q_{F}$ of $Q$ in $S_{F}$, and every local B-monoid factor of $S$ can be obtained in this manner.

Proof. Clearly, $F$ is an $m$-prime filter and $Q, K$ are filters. Assume $\varphi(x)=\varphi(y) \neq$ 0 . Then $x, y$ are not in $Q$. Let $d=x \wedge y=d\left(x_{1} \wedge y_{1}\right), x_{1} \wedge y_{1}=1, x=d x_{1}, y=d y_{1}$. The equalities $\varphi(d)=\varphi(x)=\varphi(y)=\varphi(x \vee y)=\varphi\left(d x_{1} y_{1}\right)=\varphi(d) \varphi\left(x_{1} y_{1}\right) \neq 0$ imply $\varphi\left(x_{1} y_{1}\right)=1$, and hence for $s=x_{1} y_{1} \notin F$ we have $x \leq x \vee y=y x_{1} \leq$ $y s, y \leq x \vee y=x y_{1} \leq x s$, from which the first part of the theorem follows. For the second part, it is straightforward to check that $(*)$ is a congruence of $S$, hence $S_{F}$ is a distributive $l$-monoid. For the sake of convenience we will denote by $z_{F}$ the image of $z$ in $S_{F}$. For any two $a, b \in S$ let $d=a \wedge b, a=d a_{1}, b=d b_{1}, a_{1} \wedge b_{1}=1$ with appropriate $a_{1}, b_{1} \in S$. Then at least one of $a_{1}, b_{1}$ is not contained in $F$, hence $a_{F}, b_{F}$ are comparable, i.e., $S_{F}$ is totally ordered. An equality $a_{F}=a_{F} \wedge b_{F}$ implies $a_{F}=d_{F}$ and $b_{F}=\left(d b_{1}\right)_{F}=d_{F}\left(b_{1}\right)_{F}=a_{F}\left(b_{1}\right)_{F}$, that is, $a_{F} \mid b_{F}$. Likewise, $a_{F} \mid b_{F}$, that is, $a_{F} c_{F}=b_{F}$ for some $c \in S$ means that $a c \leq b s$ and $b \leq a c s$ 
for some $s \notin F$, whence $a \leq b s$, which implies $a_{F} \leq b_{F}$. This shows that the induced order in $S_{F}$ is natural. Finally, for hyper-normality assume $a_{F} c_{F}=b_{F}$ and $a_{F} e_{F}=a_{F}$ for some $c$ and $e$ in $S$. By definition we have ae $\leq a s$ for some $s \notin F$. Then $e \wedge s=d \notin F$ holds by $d \leq s \notin F$ and $e=d u$ for some $u$. Moreover, $a d u=a e=a e \wedge a s=a(e \wedge s)=a d$. Thus, hyper-normality of $S$ implies the existence of an element $t$ such that $a d=a d u=a e \leq a c s=a d t$ and $u \wedge t=1$. Consequently we have $b_{F}=(a c)_{F}=(a c s)_{F}=(a d t)_{F}=a_{F} t_{F}$ and $(e \wedge t)_{F}=(d u \wedge t)_{F} \leq d_{F}(u \wedge t)_{F}=d_{F}=1$, which shows that $S_{F}$ is hyper-normal.

The last claim is now obvious.

Remarks 2.12. (1) For an $m$-prime filter $F$ the factor of $S$ by the congruence (*) is called the localization $S_{F}$ of $S$ at $F$ (sending $S \backslash F$ to 1 ) after the classical notion of localization at prime ideals in commutative algebra.

(2) This kind of localization or, equivalently, congruence can be defined in a more general form; see [9, Proposition 0.9].

(3) One of the main aims of this section is a description of all homomorphisms between $B$-semigroups. In doing this, we do not proceed directly. We start from a distributive $l$-semigroup congruence on the given $B$-monoid and then check that the resulting factor satisfies two conditions: naturality of the induced partial order and hyper-normality. Some related results on homomorphisms between distributive $l$-semigroups can be found in 7 and 9]. A general description of homomorphisms between $B$-semigroups will be given in Theorem 2.26 below.

Proposition 2.13. Localizations (i.e., congruences of the kind (*)) on a B-monoid $S$ permute. That is, if we denote by $\Phi_{F}$ the congruence $(*)$ belonging to an $m$-prime filter $F$, and $F, F^{\prime}$ are $m$-prime filters on $S$, then we have $\Phi_{F} \circ \Phi_{F^{\prime}}=\Phi_{F^{\prime}} \circ \Phi_{F}$, where o denotes the relational product.

Proof. Let $x \Phi_{F} y$ with $s \notin F$ such that $x \leq y s, y \leq x s$, and $y \Phi_{F^{\prime}} z$ with $t \notin F^{\prime}$ such that $y \leq z t, z \leq y t$. Put $u=x t \wedge z s$; then $x \Phi_{F^{\prime}} u$ since $u \leq x t$ is clear and $u t=x t^{2} \wedge z s t \geq x t^{2} \wedge y s \geq x t^{2} \wedge x=x$, and $u \Phi_{F} z$ since $u \leq z s$ is clear and $u s w=x t s \wedge z s^{2} \geq y t \wedge z s^{2} \geq z \wedge z s^{2}=z$.

Remark 2.14. This shows that the congruences which we will use in the Grothendieck-type sheaf representation of $B$-monoids (see Theorem 4.10) permute.

The following theorem stresses the importance of filters by marking the parallelism of certain congruences of $B$-monoids with ideals in ring theory. Although Rees factors are suitable for working with 0-cancellative naturally totally ordered semigroups, i.e., with local $B$-monoids, they are not appropriate for dealing with general $B$-monoids. Our next result makes this situation clear.

Theorem 2.15. Let $S$ be a B-monoid and $F$ be any filter. Then

$$
x \cong y \Longleftrightarrow \exists s \in F: x \wedge s=y \wedge s, x, y, \in S
$$

defines a congruence of $S$ whose factor $S / F$ is again a B-semigroup, called the factor B-monoid of $S$ by the filter $F$.

Proof. It is well known from lattice theory that $(* *)$ is a lattice congruence. Moreover, it is straightforward to see that it is indeed a congruence of $S$. The image of an arbitrary element $a \in S$ in $S / F$ will be denoted by $\bar{a}$. First, we show the 
naturality of the induced order on $S / F$. Let $\bar{a} \wedge \bar{b}=\bar{a}$; then $\bar{d}=\bar{a}$ for $d=a \wedge b$ and hence $b=d c$ for some $c$, so $\bar{b}=\overline{d c}=\bar{d} \bar{c}=\bar{a} \bar{c}$. Conversely, the equality $\bar{b}=\bar{a} \bar{c}=\overline{a c}$ for some $a, b, c \in S$ means that $b \wedge s=a c \wedge s$ for some $s \in F$. Consequently, $a \wedge s \leq b \wedge s$ implies $\bar{a} \leq \bar{b}$. This verifies the naturality of the induced order in $S / F$.

Finally, assume that hyper-normality fails for $S / F$. Then there are $a, b, e \in S$ with $\bar{a}=\bar{a} \bar{e} \leq \bar{b}$ such that there is no $x \in S$ with $\bar{b}=\bar{a} \bar{x}$ and $\bar{e} \wedge \bar{x}=1$. Since $\bar{b}=\bar{a} \bar{x}$ implies $\bar{b}=\bar{a}(\overline{x \wedge y})$ for all $y \in F: a$, this shows that $1 \notin X=[x \wedge(F: a)] \wedge e$. Therefore the filter generated by $X$ is proper, and hence it is contained in an $m$ prime filter $P$. Moreover, in the totally ordered localization $S_{P}$ of $S$ at $P$ we have that $a_{P}$ is not an element of $F_{P}$, the image of $F$ in $S_{P}$ (where $x_{P}$ denotes the image of $x \in S$ in $S_{P}$ ). For, by the definition of localization, $a_{P} \in F_{P}$ means as $\in F$ for some $s \notin P$. But as $\in F$ implies $s \in F: a \subseteq P$, a contradiction. Therefore $a_{P} \notin F_{P}$, and hence the image $\tilde{a_{P}}$ of $a_{P}$ in the Rees factor $T$ of $S_{P}$ by $F_{P}$, which is obviously a $B$-monoid, is not 0 . However, in this $T$ we have $0 \neq \tilde{a_{P}}=\tilde{a_{P}} \tilde{e_{P}}$, but $\tilde{e_{P}} \neq 1$ by $e \in P$, again a contradiction. Thus $S / F$ is hyper-normal, whence it is a $B$-monoid.

Remarks 2.16 .

(1) Let $S$ be the semigroup of divisibility of a Bezout ring $R$ and take any ideal $I$ of $R$. Then the semigroup of divisibility of $R / I$ is just $S / F$, where $F$ is the filter consisting of the principal ideals contained in $I$.

(2) Such a kind of congruence can be defined for a larger class of distributive $l$-semigroups; see [9, Proposition 0.9 ].

Proposition 2.17. Factors by filters (i.e., congruences of the kind (**)) on a Bmonoid $S$ permute. That is, if we denote by $\theta_{F}$ the congruence (**) belonging to a filter $F$, and $F, F^{\prime}$ are filters on $S$, then we have $\theta_{F} \circ \theta_{F^{\prime}}=\theta_{F^{\prime}} \circ \theta_{F}$, where $\circ$ denotes the relational product.

Proof. Let $x \theta_{F} y$ with $s \in F$ be such that $x \wedge s=y \wedge s$ and $y \theta_{F^{\prime}} z$ with $t \in F^{\prime}$ such that $y \wedge t=z \wedge t$. Put $u=(x \wedge t) \vee(z \wedge s)$. Then $x \theta_{F^{\prime}} u$ since $u \wedge t=$ $(x \wedge t) \vee(z \wedge s \wedge t)=(x \wedge t) \vee(y \wedge s \wedge t)=(x \wedge t) \vee(x \wedge s \wedge t)=(x \wedge t)$ and $u \theta_{F} z$ since $u \wedge s=(x \wedge t \wedge s) \vee(z \wedge s)=(y \wedge t \wedge s) \vee(z \wedge s)=(z \wedge t \wedge s) \vee(z \wedge s)=z \wedge s$.

Remark 2.18. This shows that the congruences which we will use in the Pierce-type sheaf representation of $B$-monoids (see Theorem 4.5) permute.

We are now in a position to discuss the lifting of idempotents modulo the nil radical. As an easy consequence of Lemma 1.11 one has:

Proposition 2.19. Let $N$ be the nil radical of a B-monoid $S$ and $\bar{e}^{2}=\bar{e}$ be an idempotent in the factor B-monoid $S / N$. Then there is an idempotent $e^{2}=e \in S$ whose image in $S / N$ is $\bar{e}$. In particular, for any finite set of pairwise orthogonal idempotents $\bar{e}_{i} \in S / N$ there are pairwise orthogonal idempotents $e_{i} \in S$ such that each $\bar{e}_{i}$ is the image of $e_{i}$.

Proof. Let $\bar{f} \in S / N$ be the complement of $\bar{e}$ in $S / N$, i.e., $\bar{f}^{2}=\bar{f}, \bar{e} \wedge \bar{f}=1, \bar{e} \bar{f}=0$ and $a, b \in S$ such that $\bar{e}, \bar{f}$ are images of $a, b$ in $S / N$, respectively. Then we have $a \wedge b \wedge c=1$ for some nilpotent element $c \in S$ and $a b \in N$. Thus there is an integer $n$ such that $0=c^{n}=(a b)^{n}=a^{n} b^{n}$. By Lemma 1.11 one has $1=a^{n} \wedge b^{n} \wedge c^{n}=$ $a^{n} \wedge b^{n}$ and $0=a^{n} b^{n}=a^{n} \vee b^{n}$. Therefore $e=a^{n}$ is the required idempotent. The other statement is now obvious. 
The next result can be compared with claim (iii) in [10, Proposition 7.7] and [32, Proposition 3.7] for rings.

Proposition 2.20. A B-monoid is a finite direct product of primary B-monoids iff it has only finitely many minimal m-prime filters.

Proof. It is enough to see only the sufficiency, which is essentially a simple case of the Chinese Remainder Theorem. Let $\left\{P_{1}, \ldots, P_{n}\right\}$ be the finite set of minimal $m$-prime filters of $S$. By Corollary 2.10 one has $P_{n} \wedge P_{j}=S$ for all $j<n$. This implies $P_{n} \wedge Q_{n}=S$ with $Q_{n}=\prod_{i=1}^{n-1} P_{i}$. Hence there are $a \in P_{n}, a_{n} \in Q_{n}$ with $a \wedge a_{n}=1$ and $a a_{n}$ nilpotent, say $\left(a a_{n}\right)^{l}=0$. For $e=a^{l}, e_{n}=a_{n}^{l}$ one has $e e_{n}=0$, $e \wedge e_{n}=1$, hence $e^{2}=e \in P_{n}, e_{n}^{2}=e_{n} \in Q_{n}$. For $S_{n}=e_{n} S, T=e S$ one can see immediately that $S_{n}$ is a primary $B$-monoid and $T$ has $n-1$ minimal $m$-prime filters. Therefore the statement follows by induction.

It follows from the above that an indecomposable $B$-monoid has either a smallest $m$-prime filter or infinitely many minimal $m$-prime filters (in fact, both cases can occur).

Localization of an arbitrary commutative ring by a (saturated multiplicatively closed) subset induces a homomorphism between the semigroups of divisibility of the considered rings. For $B$-monoids, the notion corresponding to a saturated multiplicatively closed subset is an $m$-cofilter, that is, a multiplicatively closed subset $C$ such that $a \leq b$ and $b \in C$ imply $a \in C$. Now we show that localization by an $m$-cofilter is a surjective homomorphism.

Theorem 2.21. If $C$ is an $m$-cofilter in a B-monoid $S$, then, for $x, y \in S$,

$$
x \cong y \Longleftrightarrow \exists s \in C: x \leq y s \text { and } y \leq x s
$$

defines a congruence on $S$ whose factor is a B-monoid, called the localization $S_{C}$ of $S$ at $C$, and the congruence class containing 0 is $K=\{x \in S \mid \exists s \in C: s x=0\}$.

We omit the straightforward proof because one can see this in the same way as in the second part of the proof of Theorem 2.11.

Remarks 2.22.

(1) Clearly, the congruence defined in Theorem 2.21 contains the congruence $(* *)$ defined in Theorem 2.15, and they need not be equal. See also Example 2.28

(2) For any subset $A$ of $S$, sending $A$ to 1 results in the localization $S_{C}$ of $S$ at the $m$-cofilter $C$ consisting of the divisors of finite products of elements in $A$. If an $m$-cofilter $C$ is the complement of an $m$-prime filter $F$, then, as usual, we write $S_{F}$ instead of $S_{C}$.

(3) Theorem 2.21 obviously generalizes the localization at $m$-prime filters described in Theorem 2.11. We have separated the two constructions because of the key role of Theorem 2.11. In addition, we think that this manner of presentation is easier to follow.

Proposition 2.23. If $a, x, y \in S$ in a $B$-monoid $S$, then $x a=y a$ holds if and only if there is an $s \in a^{\perp}$ such that $x \wedge s=y \wedge s$. 
Proof. Without loss of generality one can assume $x \leq y$, i.e, $y=t x$ for some $t \in S$ (we could consider $x \wedge y$ and $y$ instead of $x$ and $y$ ). Putting $b=a x$ one obtains $b t=b$. Therefore by hyper-normality there is $s \in b^{\perp}=(a x)^{\perp}$ with $t \wedge s=1$. Consequently, we have $x \wedge s x=x=x(t \wedge s)=t x \wedge s x=y \wedge s x$, from which the statement follows in view of $s x \in a^{\perp}$.

Corollary 2.24. If 0 is an m-prime filter in a B-monoid $S$, then $S$ is cancellative. Consequently, if $F$ is an $m$-prime filter of an arbitrary $B$-monoid $S$, then the factor $S / F$ is cancellative.

Remark 2.25. It is at this point that we can see the importance of hyper-normality. If we extend the complement of an $m$-prime filter of a $B$-monoid by a zero element, then we obtain a $D$-monoid which has no zero-divisors but is not hyper-normal: the condition of hyper-normality is satisfied for any triple $a, b, e$ which are all different from 0 but it does not hold if $a \neq 0$ and $b=0$.

$B$-monoids are modelled after the divisibility theory of Bezout rings, so they are somewhat simpler structures than the latter. Hence there are, as one can expect, "in some sense" more homomorphisms between them than the ones induced by homomorphisms between rings. However, combining Theorems 2.15 and 2.21, Remark 2.22, and Proposition 2.23 one can describe, as the main result of this section, all homomorphisms between $B$-monoids as follows.

Theorem 2.26. Let $\psi$ be a homomorphism of a $B$-monoid $S$ onto a $B$-monoid. Put $C=\{s \in S \mid \psi(s)=1\}$ and $F=\{s \in S \mid \psi(s)=0\}$. Then $C$ is an m-cofilter, $F$ is a filter, and for all $a \in S, s \in F$ and $c \in C, a \wedge s \leq c$ implies $a \in C$ and $s \leq$ ca implies $a \in F$. Now the equality $\psi(x)=\psi(y)$ holds if and only if there are $s \in F, c \in C$ with $y \wedge s \leq c x$ and $x \wedge s \leq c y$. In particular, $\psi(S)$ is a B-monoid, isomorphic to the factor $B$-monoid $S_{\psi}$ of the localization $S_{C}$ of $S$ by the image $F_{C}$ of $F$ in $S_{C}$.

Conversely, given an $m$-cofilter $C$ and a filter $F$ in a B-monoid $S$ such that, for all $a \in S, s \in F$ and $c \in C, a \wedge s \leq c$ implies $a \in C$ and $s \leq c a$ implies $a \in F$, then the relation

$$
\Psi=\{(x, y) \in S \times S \mid \exists s \in F, c \in C: y \wedge s \leq c x \text { and } x \wedge s \leq c y\}
$$

is a congruence of lattice-ordered semigroups whose 1-class is $C$ and 0 -class is $F$, and the factor $S / \Psi$ is a B-monoid.

Proof. Let $\psi, C, F$ be as in the first part of the statement. The claims about $C$ and $F$ are obvious. Next, let $x, y \in S$ with $\psi(x)=\psi(y)$. By the equality $\psi(x \wedge y)=\psi(x \vee y)$ one can assume without loss of generality that $x \leq y=d x$ for some $d \in S$. Therefore $\psi(x)=\psi(d) \psi(x)$ implies $\psi(d) \wedge \psi(t)=1$ for some $t \in S$ with $\psi(t) \in \psi(x)^{\perp}$ in view of Proposition 2.23. Consequently, $t x \in F$ and $c=d \wedge t \in C$ hold. Hence $y \wedge t x=d x \wedge t x=c x \leq c x$, which completes the proof of the first part because $x \wedge t x \leq x \leq y \leq c y$ is trivially satisfied.

Conversely, suppose $C$ and $F$ are given as indicated. The given relation $\Psi$ is clearly reflexive and symmetric. It is also transitive because in case $y \wedge s \leq c x$, $x \wedge s \leq c y, y \wedge t \leq d z, z \wedge t \leq d y$, putting $u=s \wedge t$ and $e=c d$ we have

$$
x \wedge u=(x \wedge s) \wedge t \leq c y \wedge t \leq c(y \wedge t) \leq c d z=e z,
$$

and similarly $z \wedge u \leq e x$. Using the distributivity laws in $S$, it is straightforward to check that $\Psi$ is a congruence relation of lattice-ordered semigroups. It is clear that 
$c \Psi 1$ for every $c \in C$ and $s \Psi 0$ for every $s \in F$. Next, if $a \Psi 1$ for an $a \in S$, then there exist $c \in C, s \in F$ such that $a \wedge s \leq c$. Hence $a \in C$ by assumption, and likewise, $a \Psi 0$ implies $a \in F$. Proving that $S / \Psi$ is a $B$-monoid proceeds in the same manner as in Theorems 2.11 and 2.15 ,

Remarks 2.27.

(1) As is easy to see, if $C$ and $F$ are as in the second part of Theorem 2.26. then $C \cap F \neq \varnothing$ implies $C=S=F$.

(2) If $C \subseteq S$ is a cofilter, then $\bar{C}=\left\{s \in S \mid \exists n \in \mathbb{N}, c_{1}, \ldots, c_{n} \in C: s \leq\right.$ $\left.c_{1} \ldots c_{n}\right\}$ is the smallest $m$-cofilter containing $C$. If $M$ is any multiplicatively closed subset of $S$, then $C=\{s \in S \mid \exists m \in M: s \leq m\}$ is the smallest $m$-cofilter containing $M$. In fact, this smallest $m$-cofilter containing $M$ is just the saturation of $M$, that is, the set of all divisors of elements of $M$.

(3) Let $C, F \subseteq S$ be an arbitrary cofilter or a multiplicatively closed subset and a filter, respectively. We want to describe the smallest congruence for which $C$ and $F$ are contained in the 1-class and the 0-class, respectively. Clearly, the 1-class of this congruence contains the smallest $m$-cofilter containing $C$, and by the first part of Theorem 2.26, the 0-class must contain the set $\{s \in S \mid \exists c \in C$ and $b \in F: b \leq c s\} \supseteq F$, hence also the smallest filter containing this set. Doing this extension first, if necessary, we can assume that $C$ is an $m$-cofilter and the filter $F$ satisfies $F \supseteq\{s \in S \mid \exists c \in C$ and $b \in F: b \leq c s\}$. Now, let $C_{1}=\{a \in S \mid \exists c \in C, s \in F: a \wedge s \leq c\}$. Then $C_{1} \supseteq C$ is also an $m$-cofilter. Let $F_{1}=\{b \in S \mid \exists s \in F, c \in C: s \leq b c\}$. Then $F_{1} \supseteq F$ is also a filter. Put $C_{2}=\left\{a \in S \mid \exists c \in C_{1}, s \in F_{1}: a \wedge s \leq c\right\}$ and $F_{2}=\left\{b \in S \mid \exists s \in F_{1}, c \in C_{1}: s \leq b c\right\}$. We show that $C_{2}=C_{1}$ and $F_{2}=F_{1}$. Indeed, if $a \in C_{2}$, then there exist $c_{1} \in C_{1}$ and $s_{1} \in F_{1}$ such that $a \wedge s_{1} \leq c_{1}$. For $c_{1} \in C_{1}$ there are $\bar{c} \in C$ and $\bar{s} \in F$ with $c_{1} \wedge \bar{s} \leq \bar{c}$, and for $s_{1} \in F_{1}$ there are $\tilde{s} \in F$ and $\tilde{c} \in C$ with $\tilde{s} \leq \tilde{c} s_{1}$. Therefore we obtain $a \wedge s_{1} \wedge \bar{s} \leq c_{1} \wedge \bar{s} \leq c$ and hence $\tilde{c}\left(a \wedge s_{1} \wedge \bar{s}\right)=\tilde{c} a \wedge \tilde{c} s_{1} \wedge \tilde{c} \bar{s} \geq \tilde{c} a \wedge \tilde{s} \wedge \tilde{c} \bar{s}$ consequently, $\tilde{c} a \wedge(\tilde{s} \wedge \tilde{c} \bar{s}) \leq \tilde{c} \bar{c}$. Now $\bar{s} \leq(\tilde{c} \bar{s}) 1$; hence $\tilde{c} \bar{s} \in F$ by our assumption, and $\tilde{c} \bar{c} \in C$, so $\tilde{c} a \in C_{1}$ and thus, in view of $a \leq \tilde{c} a$, we have $a \in C_{1}$. Similarly, if $b \in F_{2}$, then there exist $s_{1} \in F_{1}$ and $c_{1} \in C_{1}$ such that $s_{1} \leq b c_{1}$. For $c_{1}$ there are $\bar{c} \in C$ and $\bar{s} \in F$ with $c_{1} \wedge \bar{s} \leq \bar{c}$ and for $s_{1}$ there are $\tilde{s} \in F$ and $\tilde{c} \in C$ with $\tilde{s} \leq \tilde{c} s_{1}$. So we have $s_{1} \wedge b \bar{s} \leq$ $b c_{1} \wedge b \bar{s}=b\left(c_{1} \wedge \bar{s}\right) \leq b \bar{c}$, and then $\tilde{s} \wedge \tilde{c} b \bar{s} \leq \tilde{c} s_{1} \wedge \tilde{c} b \bar{s} \leq b \bar{c} \tilde{c}$. Here $\bar{c} \tilde{c} \in \bar{C}$ and $\tilde{s} \wedge \tilde{c} b \bar{s} \in F$; hence by the assumption $b \in F_{1}$. So we have $C_{2}=C_{1}$ and $F_{2}=F_{1}$, as claimed. Thus the $m$-cofilter $C_{1}$ and the filter $F_{1}$ satisfy the conditions in the converse part of Theorem 2.26. The congruence they determine is clearly the smallest congruence for which $C$ is in the 1-class and $F$ is in the 0 -class.

(4) Contrary to the special cases of congruences of types $(*)$ and $(* *)$ (see Remarks 2.13 and 2.17), we are not able to prove that arbitrary congruences (that is, those of type $\Psi$ ) permute.

Example 2.28. The following simple example shows that $m$-cofilters $C$ are in general necessary for describing homomorphisms between $B$-monoids. Let $\mathfrak{P}$ be the semigroup of divisibility of the Bezout ring $R=\mathbb{Z} \ltimes \mathbb{Q} / \mathbb{Z}$, the trivial extension of $\mathbb{Z}$ by the Abelian group $\mathbb{Q} / \mathbb{Z}$. Thus $\mathfrak{P}$ is generated by $s_{p}, t_{p^{-n}} ; n \in \mathbb{N}, p \in$ 
$\mathbb{P}(=$ prime numbers $)$ subject to $s_{p} t_{p}=t_{p} t_{q}=0$ for all $p, q \in \mathbb{P}$ and $s_{p} t_{p^{-n}}=$ $t_{p^{n-1}}, s_{p} t_{q}=t_{q}$ for all $p \neq q \in \mathbb{P} ; n>1$. Then $\mathfrak{P}$ is a $B$-monoid with one minimal $m$-prime filter $M$ generated by all $t_{p^{-n}}$, and every element of $\mathfrak{P}$ different from 0 and 1 is a zero-divisor. Thus, if we consider the homomorphism of $\mathfrak{P}$ into the (indecomposable) two-element Boolean algebra by sending $M$ to 0 and its complements to 1 , then $F=M$ and $C=\mathfrak{P} \backslash M$. Hence the factor of $S$ by $F$ is just the multiplicative semigroup $\mathbb{N}$ of natural numbers, which is obviously not the two-element Boolean algebra.

In what follows, we give a short description of classical localizations of $B$-monoids where the localization is not a $B$-monoid. For any multiplicatively closed subset $M$ of a $B$-monoid $S$ one can define the classical localization $S_{M}$ of $S$ at $M$ as follows. $S_{M}$ is the set of equivalent classes of pairs $(a, s)$ with $a \in M, s \in S$ with respect to the relation $(a, s) \sim\left(a_{1}, s_{1}\right)$ if there exists $a_{2} \in M$ with $a_{2} a_{1} s=a_{2} a s_{1}$. Then $S_{M}$ will be a hyper-normal distributive $l$-monoid by putting

$$
(a, s)\left(a_{1}, s_{1}\right)=\left(a a_{1}, s s_{1}\right)
$$

and

$$
(a, s) \wedge\left(a_{1}, s_{1}\right)=\left(a a_{1}, s a_{1} \wedge a s_{1}\right),(a, s) \vee\left(a_{1}, s_{1}\right)=\left(a a_{1}, s a_{1} \vee a s_{1}\right)
$$

We write $a^{-1} s$ for the equivalence class of $(a, s)$. It is a tedious but routine task to check that this definition is independent of the choice of representatives and then to verify all the axioms required by the definition of distributive $l$-monoids. Note that 1 is not, in general, multiplicatively irreducible, that is, $S_{M}$ need not be a $B$-monoid. Hyper-normality of $S_{M}$, however, follows immediately from the observation that $a^{-1} s \leq b^{-1} t$ if and only if $b^{-1} t=a^{-1} s u$ for some $u \in S$. Indeed, the condition implies $a^{-1} s=a^{-1} s \wedge b^{-1} t=(a b)^{-1}(s b \wedge a t)$. Hence there exists $c \in M$ with $c a b s=c a(s b \wedge a t) \leq c a^{2} t$; consequently there is $u \in S$ with $c a b s u=c a^{2} t$, whence $a^{-1} s u=\left(a^{2} b c\right)^{-1} c a b s u=\left(c a^{2} b\right)^{-1} c a^{2} t=b^{-1} t$. There is in addition a natural homomorphism from $S$ into $S_{M}$, which takes all elements of $S$ annihilated by some $a \in M$ to $0 \in S_{M}$. Therefore the filter $K$ generated by these elements goes to $0 \in S_{M}$. The image of $S$ in $S_{M}$ is precisely the factor of $S$ by $K$ described in Theorem 2.15, hence it is a $B$-monoid. Moreover, the image of $S$ in $S_{M}$ is exactly the subsemigroup of the positive elements of $S_{M}$. This leads to the following interesting result.

Proposition 2.29. For an m-prime filter $I$ of a B-monoid $S$, put $K=\{x \in$ $S \mid \exists s \notin I: s x=0\}$. Then $K$ is a filter, and the factor $S / K$ has a unique minimal $m$-prime filter, which is the image of the smallest m-prime filter contained in $I$.

Proof. It is easy to see that $K$ is a filter. We denote the image of $x \in S$ in $S / K$ by $\bar{x}$. To verify the statement it is enough to see that the nil radical is an $m$-prime filter. For this aim, assume indirectly that, for some $a, b \in S$, the element $\overline{a b}$ is nilpotent but $\bar{a}$ and $\bar{b}$ are not. This implies $(a b)^{n} \in K$ for some $n \in \mathbb{N}$. Therefore $a_{I} b_{I}$ is nilpotent, where $x_{I}$ denotes the image of $x \in S$ in the localization $S_{I}$ of $S$. Since $S_{I}$ is a totally ordered $B$-monoid, its nil radical is an $m$-prime filter. Consequently, one of $a_{I}$ and $b_{I}$ is nilpotent, hence, by the definition of $K$, so is one of $\bar{a}$ and $\bar{b}$, a contradiction.

This result has an interesting consequence for arithmetical rings, taking into consideration that the (prime) ideals of an arithmetical ring $R$ are exactly the ( $m$-prime) filters of the divisibility monoid of $R$. 
Corollary 2.30. Let $I$ be a prime ideal in an arithmetical ring $R$ and $K$ be the set of annihilators of elements not contained in $I$. Then $K$ is an ideal and the factor ring $R / K$ has only one minimal prime ideal.

\section{SEMIPRIMe $B$-MONOIDS}

In this section we describe the structure of semiprime $B$-monoids. Unusual examples for semiprime $B$-monoids are semigroups of divisibility of certain rings of continuous functions; see [18.

Proposition 3.1. If $S$ is a semiprime B-monoid, then, for every element $a \in S$,

$$
a^{\perp}=\{x \in S \mid x a=0\}=\{x \in S \mid x \vee a=0\} .
$$

Proof. $0=x \vee a \leq x a$ trivially implies $x a=0$. Conversely, $0=x a \leq(x \vee a)^{2}$, whence $0=(x \vee a)^{2}=x \vee a$ by the semiprimeness of $S$.

Proposition 3.2. If $S$ is a semiprime B-monoid, then for every element $a \in S$, $a^{\perp}$ is an m-semiprime filter, i.e, $a^{\perp}=I\left(a^{\perp}\right)$.

Proof. If $x^{n} \in a^{\perp}$, then we have $(a x)^{n}=0$, whence $a x=0$ and therefore $x \in a^{\perp}$, from which the statement follows.

Proposition 3.3. If $S$ is a semiprime B-monoid, then for any $a, b \in S$ it holds that

$$
(a b)^{\perp}=(a \vee b)^{\perp},(a \wedge b)^{\perp}=a^{\perp} \cap b^{\perp} .
$$

Proof. $s(a b)=0$ implies $(s(a \vee b))^{2} \geq s(a \vee b)^{2} \geq s a b=0$, whence $s(a \vee b)=0$, i.e., $(a b)^{\perp}=(a \vee b)^{\perp}$. Since $x(a \wedge b)=x a \wedge x b=0 \Leftrightarrow x a=0=x b$, we obtain $(a \wedge b)^{\perp}=a^{\perp} \cap b^{\perp}$.

For an element $a \in S$, put $S_{a}=\left\{b \in S \mid a^{\perp}=b^{\perp}\right\}$.

Proposition 3.4. Let $S$ be a semiprime B-monoid. For $x, y \in S_{a}$ and $b \in S$ with $b^{\perp} \subseteq a^{\perp}$ the equality $0 \neq b x=b y$ implies $x=y$.

Proof. The assumption implies $a, b, x, y \neq 0$. One can assume, without loss of generality, that $x \leq y=x d$ for some $d \in S$. Assume indirectly that $d \neq 1$. We have $z=b x=b y=b x d=z d \neq 0$. Moreover, if $s(b x)=0$, then $s x \in b^{\perp} \subseteq a^{\perp}=x^{\perp}$ implies $(s x)^{2}=0$. Hence $s x=0$, i.e., $(b x)^{\perp}=x^{\perp}=a^{\perp}$. This implies that there is no $u \in z^{\perp}$ with $d \wedge u=1$, otherwise we would have $x=x(d \wedge u)=x d=y$, a contradiction. Hence there is an $m$-prime filter $F$ containing $d \wedge x^{\perp}$. Thus in the localization $S_{F}$ we have $d_{F} \neq 1$ and $0 \neq z_{F}=z_{F} d_{F}$, a contradiction.

Theorem 3.5. If $S$ is a semiprime $B$-monoid, then, for every element $a \in S$, the subsemigroup $S_{a}$ is a cancellative semigroup closed under meet and join. Recall that the factor $S / a^{\perp}$ of $S$ comes from the congruence

$$
x \cong_{a} y \Longleftrightarrow \exists s \in a^{\perp}: x \wedge s=y \wedge s, x, y \in S .
$$

Then $S_{a}$ maps injectively into $\left(S / a^{\perp}\right)_{1}$, and these two semigroups have the same group of quotients. Moreover, $S_{a}^{\bullet}$ is a B-monoid if and only if its induced order is natural, i.e., $b \leq c$ in $S_{a}$ implies $c=b u$ for some $u \in S_{a}$.

Thus a semiprime $B$-monoid $S$ is the disjoint union of cancellative semigroups $S_{a}, a \in S$, where $S_{0}=0$ and $S_{1}$ is the semigroup of non-zero-divisors. 
Proof. Assume $x, y \in S_{a}$. Then $(x \wedge y)^{\perp}=x^{\perp} \cap y^{\perp}=a^{\perp}$. If $t(x \vee y)=0$, then $t x y=0$. Hence $t x \in y^{\perp}=x^{\perp}$, thus $(t x)^{2}=0 \Longrightarrow t x=0$, i.e. $t \in x^{\perp}$, and this also shows that $x y \in S_{a}$. Thus $S_{a}$ is a subsemigroup closed under both $\wedge$ and $\vee$. If now $x z=y z$ with $x, y, z \in S_{a}$, then, in view of $(x \vee y) z=x z \vee y z=x z$, one can assume that $y=d x$, and hence we have $z x=d z x$. Thus $x \neq y$ would imply $d \neq 1$. If there is some $c \in x^{\perp}=a^{\perp}$ with $c \wedge d=1$, then we have $x=x \cdot 1=x(c \wedge d)=x d=y$, a contradiction. Thus there is an $m$-prime filter $F$ such that in the 0 -cancellative totally ordered localization $S_{F}$ we have $d_{F} \neq 1,(x z)_{F} \neq 0$ in spite of the equality $(x z)_{F}=d_{F}(x z)_{F}$, a contradiction. By Theorem [2.15, $\cong_{a}$ is a lattice-orderedsemigroup congruence.

By definition, any $x \in S_{a}$ obviously maps to a non-zero-divisor in $S / a^{\perp}$. Furthermore, $S_{a}$ is embedded in $S / a^{\perp}$. For $b, c \in S_{a}$ with $b \wedge x=c \wedge x, x \in a^{\perp}$ we have $b^{2}=b(b \wedge x)=b(c \wedge x)=b c$, and hence $b=c$ by the previous argument. To show that $S_{a}$ and $\left(S / a^{\perp}\right)_{1}$ have the same group of quotients, it is enough to see that $\left(S / a^{\perp}\right)_{1}$ is contained in the quotient group of $S_{a}$. For this aim, assume $b \in S$ maps to a non-zero-divisor in $S / a^{\perp}$. This means precisely that $b x \in a^{\perp}$ implies $x \in a^{\perp}$. Therefore $a x \in a^{\perp}$ follows from the equality $x a b=0 \in a^{\perp}$, hence $(a x)^{2}=0$. Thus $a x=0$ holds because $S$ is semiprime. Consequently $x \in a^{\perp}$, whence we obtain $a b \in S_{a}$. Thus the image of $b$ in $S / a^{\perp}$ is contained in the quotient group of $S_{a}$ because both $a$ and $a b$ are elements of $S_{a}$.

Now assume that $S_{a}^{\bullet}$ is a $B$-monoid and $b, c \in S_{a}, b \leq c$. Then $S_{a}$ has an identity, i.e, there is an idempotent $e \in S_{a}$ and we have $c=b d$ for some $d \in S$. This implies $c=b d=b e d=b u$ with $u=d e \in S_{a}$. Conversely, $a \leq a$ implies that $a=a e$ for some $e \in S_{a}$, and hence $e=e^{2}$ is the identity of $S_{a}$, from which it follows easily that $S_{a}^{\bullet}$ is a $B$-monoid.

Corollary 3.6. Let $S$ be a semiprime B-monoid. For any two elements $u, v \in S_{a}$ and $x, y \in S_{b}$, where $a, b \in S$ are arbitrary, we have $(u x)^{\perp}=(v y)^{\perp}$.

Proof. We have

$$
s \in(u x)^{\perp} \Leftrightarrow s u \in x^{\perp}=y^{\perp} \Leftrightarrow s \in(u y)^{\perp},
$$

and hence $(u x)^{\perp}=(u y)^{\perp}$. Consequently we have

$$
(u x)^{\perp}=(u y)^{\perp}=(y v)^{\perp} .
$$

\section{Sheaf Representations of Bezout monoids}

For commutative rings there are two very important representation theorems in terms of sheaves of 'simpler' rings:

- Pierce's representation theorem of rings as sheaves of indecomposable rings on the Boolean space of its idempotents [34]. (This result applies to noncommutative rings as well using central idempotents, but then the stalks may not remain indecomposable.)

- Grothendieck's representation theorem of rings as sheaves of local rings on the Zariski space of its prime ideals [20.

In this final section, we will show that, using the techniques and results developed so far in this paper, both of these representations have analogues for $B$-monoids; these are our Theorems 4.5 and 4.10 
We will start with the Pierce sheaf. We note that Pierce's construction has been adapted to universal algebras by Keimel [28, Satz 1] and that this general result applies to $B$-monoids. However, verifying that the conditions of Keimel's theorem are satisfied for $B$-monoids does not save much effort compared to the direct proof presented below.

For the reader's sake we reproduce here the definition of a sheaf, presented in the case of a sheaf of $B$-monoids, together with some elementary properties. In doing so, we follow the presentation for rings as described by Pierce 34.

Definition 4.1. For each element $\mu \in X$ of a topological space $X$ let $S_{\mu}$ be a $B$-monoid. Assume that $S_{\mu} \cap S_{\nu}=\varnothing$ for $\mu \neq \nu$. Let $\underline{S}=\bigcup_{\mu \in X} S_{\mu}$. The "index map" $\pi: \underline{S} \longrightarrow X$ is the canonical projection sending all elements of each $S_{\mu}$ to $\mu$. Assume that a topology is imposed on $\underline{S}$ such that the following axioms are satisfied:

(1) If $s \in \underline{S}$, there exist open sets $U$ in $\underline{S}$ with $s \in U$ and $N$ in $X$ such that $\pi$ maps $U$ homeomorphically onto $N$.

(2) Let $\underline{S}+\underline{S}$ denote $\{(s, t) \mid \pi(s)=\pi(t)\}$, with the topology induced by the product topology in $\underline{S} \times \underline{S}$. Then the mappings $(s, t) \mapsto s t,(s, t) \mapsto s \wedge t$, and $(s, t) \mapsto s \vee t$ are continuous on $\underline{S}+\underline{S}$ to $\underline{S}$.

(3) The mappings $\mu \mapsto 1_{\mu}$ and $\mu \mapsto 0_{\mu}$ are continuous on $X$ to $\underline{S}$.

With these conditions the pair $(X, \underline{S})$, or just $\underline{S}$ for short, is called a sheaf of $B$ monoids over $X$. The $B$-monoids $S_{\mu}$ are called the stalks of the sheaf $\underline{S}$. For an arbitrary subspace $Y$ of $X$, a section of $\underline{S}$ over $Y$ is a continuous map $\sigma$ of $Y$ to $\underline{S}$ such that $\pi(\sigma(\mu))=\mu$ for all $\mu \in Y$. The collection of all sections of $\underline{S}$ over $Y$ is denoted by $\Gamma(Y, \underline{S})$.

Lemma 4.2. (1) The map $\pi$ of $\underline{S}$ to $X$ is open and continuous.

(2) $\Gamma(Y, \underline{S})$ is a B-monoid with a multiplication defined by

$$
\left(\sigma_{1} \sigma_{2}\right)(\mu)=\sigma_{1}(\mu) \sigma_{2}(\mu), 0(\mu)=0_{\mu}, 1(\mu)=1_{\mu}
$$

and with lattice operations defined by putting

$$
\left(\sigma_{1} \wedge \sigma_{2}\right)(\mu)=\sigma_{1}(\mu) \wedge \sigma_{2}(\mu),\left(\sigma_{1} \vee \sigma_{2}\right)(\mu)=\sigma_{1}(\mu) \vee \sigma_{2}(\mu) .
$$

(3) For any $s \in S_{\mu}$ there is a neighbourhood $N$ of $\mu$ and $\sigma \in \Gamma(N, \underline{S})$ such that $\sigma(\mu)=s$.

(4) The sets of the form $\sigma(N)$, where $N$ is open in $X$ and $\sigma \in \Gamma(N, \underline{S})$, form a basis for the open sets in $\underline{S}$.

(5) Let $\mu \in Y \subseteq X$ and $\sigma_{1}, \sigma_{2} \in \Gamma(Y, \underline{S})$ with $\sigma_{1}(\mu)=\sigma_{2}(\mu)$. Then there is a neighbourhood $N$ of $\mu$ in $X$ such that $\sigma_{1}(\nu)=\sigma_{2}(\nu)$ for all $\nu \in N \cap Y$.

Proof. Claim (1) is obvious. The verification of claim (2) is routine, though tedious. Claim (3) is an immediate consequence of Definition 4.1(1). For claim (4) it is enough to see that for any element $s$ of an open subset $U$ of $\underline{S}$ there is an open subset $N$ of $X$ and $\sigma \in \Gamma(N, \underline{S})$ with $s \in \sigma(N) \subseteq U$, which is precisely the content of claim (3). For claim (5) one observes that by Definition 4.1)(1) there are open subsets $U \ni s=\sigma_{1}(\mu)=s_{2}(\mu)$ of $\underline{S}$ and $N \ni \mu$ of $X$ such that $\pi$ is homeomorphic between $U$ and $N$, in particular one-to-one between them. Hence claim (5) follows in view of the equality $\pi\left(\sigma_{1}(\nu)\right)=\pi\left(\sigma_{2}(\nu)\right)$ for all $\nu \in N \cap Y$.

Let $S$ be a $B$-monoid. Then, by Proposition 1.14, the idempotents of $S$ form a Boolean algebra $B(S)$. Denote by $\mathcal{X}(S)$ the set of the maximal filters of $B(S)$. 
Note that in $B(S)$ maximal filters are precisely maximal ideals if we consider $B(S)$ as a ring, and Boolean rings are of dimension 1, i.e., maximal ideals are at the same time minimal prime ideals, too. We shall denote the complement of an idempotent $e$ in $B(S)$ by $e^{\prime}$.

For every $\mu \in \mathcal{X}(S)$, let $F_{\mu}$ be the filter generated by the idempotents contained in $\mu$ and put $\bar{S}_{\mu}=S / F_{\mu}$. Then $B\left(\bar{S}_{\mu}\right)=\{0,1\}$ since, for any idempotent $e$ in $S$, the pair $\left\{e, e^{\prime}\right\}$ is mapped onto $\{0,1\}$ when taking the factor by $F_{\mu}$.

To avoid confusion, we shall denote by $0_{\mu}$ and $1_{\mu}$ the zero and the identity elements of $S_{\mu}$, respectively.

Proposition 4.3. The canonical map

$$
S \longrightarrow \prod_{\mu \in \mathcal{X}(S)} \bar{S}_{\mu}: s \mapsto \sigma_{s},
$$

where $\sigma_{s}(\mu)=\bar{s}_{\mu}$ for every $\mu$, is injective. In other words, $S$ is a subdirect product of the $\bar{S}_{\mu}, \mu \in \mathcal{X}(S)$.

Proof. Take any two different elements $s, t \in S$; then $F=\{e \in B(S) \mid s e=t e\}$ is a proper filter in $B(S)$. So $F$ is contained in a maximal filter $\nu \in \mathcal{X}(S)$. If $\bar{s}_{\nu}=\bar{t}_{\nu}$ in $\bar{S}_{\nu}$, then there is an $f \in \nu$ such that $s f^{\prime}=t f^{\prime}$, hence $f^{\prime} \in F \subseteq \nu$, and then $1=f \wedge f^{\prime} \in \nu$, a contradiction. Hence $\bar{s}_{\nu} \neq \bar{t}_{\nu}$, whence $\sigma_{s} \neq \sigma_{t}$.

As usual, we endow $\mathcal{X}(S)$ with the hull-kernel topology introduced by Stone 39: the closure of a subset $\mathcal{A} \subseteq \mathcal{X}(S)$ is $\left\{\mu \in \mathcal{X}(S) \mid \mu \supseteq \bigcap_{\nu \in \mathcal{A}} \nu\right\}$. This topology makes $\mathcal{X}(S)$ a Boolean space, that is, a compact, totally disconnected Hausdorff space. The sets $\mathcal{N}(e)=\{\mu \in \mathcal{X}(S) \mid e \notin \mu\}$, for all $e \in B(S)$, are exactly the open and closed sets in $\mathcal{X}(S)$, and they form a basis for the topology.

We are now going to define a topology on the set $\mathcal{S}=\bigcup_{\mu \in \mathcal{X}(S)} \bar{S}_{\mu}$ associated to a $B$-monoid $S$ such that the functions $\sigma_{s}: \mathcal{X}(S) \longrightarrow \mathcal{S}: \mu \mapsto \bar{s}_{\mu} \in \bar{S}_{\mu} \subseteq \mathcal{S}, s \in S$, will be exactly all those continuous maps from $\mathcal{X}(S)$ to $\mathcal{S}$ which satisfy $\pi\left(\sigma_{s}(\mu)\right)=\mu$ for all $\mu \in \mathcal{X}(S)$, or equivalently, precisely all the global sections.

Lemma 4.4. Let $s, t \in S$ and $\mu \in \mathcal{X}(S)$ be such that $\sigma_{s}(\mu)=\sigma_{t}(\mu)$, that is, $\bar{s}_{\mu}=\bar{t}_{\mu}$. Then there is an $e \in B(S)$ such that $\mu \in \mathcal{N}(e)$ and $\sigma_{s}(\nu)=\sigma_{t}(\nu)$ for all $\nu \in \mathcal{N}(e)$.

Proof. By $\bar{s}_{\mu}=\bar{t}_{\mu}$, there is an $f \in \mu$ such that $s f^{\prime}=t f^{\prime}$. Put $e=f^{\prime}$; then $e \notin \mu$, hence $\mu \in \mathcal{N}(e)$, and for every $\nu \in \mathcal{N}(e)$ we have $e \notin \nu$. Consequently, $f=e^{\prime} \in \nu$, and then $s f^{\prime}=t f^{\prime}$ yields $\bar{s}_{\nu}=\bar{t}_{\nu}$, that is, $\sigma_{s}(\nu)=\sigma_{t}(\nu)$.

Theorem 4.5. Let the situation be as described above. Then the sets $\sigma_{s}(\mathcal{N}(e))$, $s \in S$, e $\in B(S)$, form an open basis for a topology on $\mathcal{S}$. If we endow $\mathcal{S}$ with this topology, then $(\mathcal{X}(S), \mathcal{S})$ is a sheaf such that $s \mapsto \sigma_{s}$ is an isomorphism between $S$ and $\Gamma(\mathcal{X}(S), \mathcal{S})$. Hence every $B$-monoid is isomorphic to the B-monoid of global sections of a sheaf of directly indecomposable B-monoids.

Proof. Suppose that $\sigma_{t}(\mathcal{N}(e)) \cap \sigma_{u}(\mathcal{N}(f))$ is not empty for some $t, u \in S ; e, f \in$ $B(S)$, i.e., there are $\mu \in \mathcal{N}(e)$ and $\nu \in \mathcal{N}(f)$ with $\bar{S}_{\mu} \ni \sigma_{t}(\mu)=\sigma_{u}(\nu) \in \bar{S}_{\nu}$. Therefore $\mu=\nu \in \mathcal{N}(e) \cap \mathcal{N}(f)$ with $\bar{t}_{\mu}=\bar{u}_{\mu}$. Then, by Lemma 4.4, there is a $g \in$ $B(S)$ such that $\sigma_{t}(\nu)=\sigma_{u}(\nu)$ for all $\nu \in \mathcal{N}(g)$. Now $\mathcal{N}(g e f) \subseteq \mathcal{N}(e) \cap \mathcal{N}(f) \cap \mathcal{N}(g)$ and $\sigma_{t}(\nu)=\sigma_{u}(\nu)$ for all $\nu \in \mathcal{N}($ gef $)$, hence $\sigma_{t}(\mathcal{N}(g e f)) \subseteq \sigma_{t}(\mathcal{N}(e)) \cap \sigma_{u}(\mathcal{N}(f))$. Thus the $\sigma_{s}(\mathcal{N}(e))$ form a basis for a topology on $\mathcal{S}$, as claimed. 
Let $\underline{s} \in \mathcal{S}$, i.e., $\underline{s}=\bar{s}_{\mu}$ for some $\mu \in \mathcal{X}(S)$ and $s \in S$. This implies $\sigma_{s}(\mu)=$ $\bar{s}_{\mu}=\underline{s} \in \bar{S}_{\mu} \subseteq \mathcal{S}$ and $\pi(\underline{s})=\pi\left(\sigma_{s}(\mu)\right)=\mu \in \mathcal{X}(S)$. Let $e \in B(S) \backslash \mu$. Then $\underline{s} \in \sigma_{s}(\mathcal{N}(e))$. Next, for any $\nu \in \mathcal{N}(e), \pi\left(\sigma_{s}(\nu)\right)=\nu$, and therefore $\pi$ maps $\sigma_{s}(\mathcal{N}(e))$ homeomorphically to $\mathcal{N}(e)$.

First we prove the continuity of the map $0: \mathcal{X}(S) \longrightarrow \mathcal{S}: \mu \mapsto 0_{\mu}$. Let $\sigma_{s}(\mathcal{N}(e))$ be an open set containing $0_{\mu}$ in $\mathcal{S}$ homeomorphic to $\mathcal{N}(e)$. Then $\sigma_{s}(\mu)=0_{\mu}$ implies es $=\left(e^{\prime}\right)^{\prime} s=0$. Consequently, $\bar{s}_{\nu}=\overline{0}_{\nu}$ for all $\nu \in \mathcal{N}(e)$ by the definition of $\mathcal{N}(e)$ because $e^{\prime} \in \nu$ and $e s=\left(e^{\prime}\right)^{\prime} s=0$. Thus $0: \mathcal{X}(S) \longrightarrow \mathcal{S}: \mu \mapsto 0_{\mu}$ is a continuous function.

Similarly, one can check as a routine exercise that $(\mathcal{X}(S), \mathcal{S})$ is indeed a sheaf. If $\sigma \in \Gamma(\mathcal{X}(S), \mathcal{S})$, then by the continuity of $\sigma$ as well as the partition of unity, there are pairwise orthogonal idempotents $e_{i} \in S$ together with elements $s_{i} \in S ; i=$ $1, \ldots, n$ such that $\mathcal{X}(S)$ is the disjoint union of $\mathcal{N}\left(e_{i}\right)$ and the restrictions of $\sigma$ and $\sigma_{s_{i}}$ agree on $\mathcal{N}\left(e_{i}\right)$. This implies $\sigma=\sigma_{s}$ for $s=\bigwedge_{i=1}^{n} s_{i} e_{i}=s \in S$.

In the second part of this section, we show that every $B$-monoid can be represented as a $B$-monoid of global sections of a sheaf of local $B$-monoids (which are 0 -cancellative naturally totally ordered $B$-monoids by Proposition 1.12), transferring Grothendieck's result [20] representing a ring as a ring of global sections of a sheaf of local rings. The idea of the proof is essentially the same as that of the standard proof for rings. Here again we note that this representation theorem may conceivably follow from very general results of Wolf, formulated in a universal algebraic language (cf. 41, Corollary 3.5], which is applicable in view of Proposition 2.17).

Denote by $\mathcal{Y}(S)$ the set of all $m$-prime filters of a $B$-monoid $S$. The so-called Zariski topology of $\mathcal{Y}(S)$ has $\{F \in \mathcal{Y}(S) \mid X \subseteq F\}$ as closed subsets where $X$ runs over subsets of $S$. The sets $\mathcal{N}(s)=\{F \in \mathcal{Y}(S) \mid s \notin F\}$ for all $s \in S$ form a basis for this topology.

We denote by $\tilde{\mathcal{S}}$ the disjoint union of the sets $S_{F}, F \in \mathcal{Y}(S)$, defined in Theorem 2.11 and, as usual, $\pi: \tilde{\mathcal{S}} \longrightarrow \mathcal{Y}(S)$ is the "index map" sending all elements of each $S_{F}$ to $F$, and, finally, for every $s \in S$, we denote by $\rho_{s}$ the function $\rho_{s}: \mathcal{Y}(S) \rightarrow \tilde{\mathcal{S}}$ : $F \in \mathcal{Y}(S) \longmapsto \rho_{s}(F)=s_{F} \in S_{F} \subseteq \tilde{\mathcal{S}}$.

As a preparation to our second sheaf representation, recall the following result of Bosbach.

Proposition 4.6 (cf. Bosbach [9, Theorem 4.4]). The canonical map

$$
S \longrightarrow \prod_{F \in \mathcal{Y}(S)} S_{F}: s \mapsto \rho_{s},
$$

where $\rho_{s}(F)=s_{F}$ for every $F$, is injective. In other words, $S$ is a subdirect product of the $S_{F}, F \in \mathcal{Y}(S)$.

Remark 4.7. By Theorem 2.11, $S_{F}$ is a totally ordered $B$-monoid, and totally ordered $B$-monoids can be represented as semigroups of divisibility of valuation rings (see [35]). Therefore every $B$-monoid can be embedded into the semigroup of divisibility of a product of valuation rings. This was already noted by Bosbach a long time ago (oral communication).

We are going to define a topology on $\tilde{\mathcal{S}}$ such that the above defined functions $\rho_{s}, s \in S$, will be exactly the continuous maps from $\mathcal{Y}(S)$ to $\tilde{\mathcal{S}}$ which satisfy 
$\pi\left(\rho_{s}(F)\right)=F$ for all $s \in S, F \in \mathcal{Y}(S)$, or equivalently, all the global sections of the sheaf $\Gamma(\mathcal{Y}(S), \tilde{\mathcal{S}})$.

Lemma 4.8. Let $s, t \in S$ and $F \in \mathcal{Y}(S)$ be such that $\rho_{s}(F)=\rho_{t}(F)$, that is, $s_{F}=t_{F}$. Then there is $a \in S$ such that $F \in \mathcal{N}(a)$, i.e., $a \notin F$, and for all $G \in \mathcal{N}(a)$ the equality $\rho_{s}(G)=\rho_{t}(G)$ holds.

Proof. The equality $s_{F}=t_{F}$ implies the existence of $a \notin F$ such that $s \leq a t$, $t \leq a s$, from which the assertion follows.

Lemma 4.9. Let the notation be as above. Then $\rho_{x}=\rho_{y}$ on $\mathcal{N}(c)$, where $x, y, c \in S$ if and only if there is an $n \in \mathbb{N}$ with $x \leq c^{n} y, y \leq c^{n} x$.

Proof. Put $a=x \wedge y, b=x \vee y$. Then there is an element $u \in S$ with $b=a u$. The equality $\rho_{x}=\rho_{y}$ on $\mathcal{N}(c)$ implies $\rho_{a}=\rho_{b}$ on $\mathcal{N}(c)$, and hence $\rho_{u}=\rho_{1}$ on $\mathcal{N}(c)$. If there is no $n \in \mathbb{N}$ with $u \leq c^{n}$, then the set $I=\left\{s \in S \mid \exists n \in \mathbb{N}: s \leq c^{n}\right\}$ is closed under $\vee$ and is disjoint from the filter $\{x \in S \mid x \geq u\}$. Therefore one can extend this filter to an $m$-prime filter $F$ disjoint from $I$. Since $c \notin F, F \in \mathcal{N}(c)$ and $u \in F$ implies $\rho_{u}(F) \neq 1=\rho_{1}(F)$, a contradiction. Consequently, there is an $n \in \mathbb{N}$ with $u \leq c^{n}$ and hence $x \leq b=a u \leq y u \leq y c^{n}$, and similarly $y \leq c^{n} x$.

We are now able to present the second main result of this section.

Theorem 4.10. The sets $\rho_{s}(\mathcal{N}(a)), s \in S, a \in S$, form an open basis for a topology on $\tilde{\mathcal{S}}$. If we endow $\tilde{\mathcal{S}}$ with this topology, then $(\mathcal{Y}(S), \tilde{\mathcal{S}})$ is a sheaf such that $s \mapsto \rho_{s}$ is an isomorphism between $S$ and $\Gamma(\mathcal{Y}(S), \tilde{\mathcal{S}})$. Hence every $B$-monoid is isomorphic to the B-monoid of global sections of a sheaf of local B-monoids, that is to say, of 0 -cancellative naturally totally ordered B-monoids.

Proof. One can check as a routine exercise that $(\mathcal{Y}(S), \tilde{\mathcal{S}})$ is indeed a sheaf. If $t \in \Gamma(\mathcal{Y}(S), \tilde{\mathcal{S}})$, then by the continuity of $t$ as well as the compactness of $\mathcal{Y}(S)$ there are elements $a_{i} \in S$ together with elements $s_{i} \in S, i=1, \ldots, n$, such that $\mathcal{X}(S)$ is the union of $\mathcal{N}\left(a_{i}\right)$ and the restrictions of $t$ and $\rho_{s_{i}}$ agree on $\mathcal{N}\left(a_{i}\right)$. This implies $\bigwedge_{i=1}^{n} a_{i}=1$ and $\rho_{s_{i}}$ agrees with $\rho_{s_{j}}$ on $\mathcal{N}\left(a_{i}\right) \cap \mathcal{N}\left(a_{j}\right)=\mathcal{N}\left(a_{i} a_{j}\right)$ for all $i, j$. Now, by Lemma 4.9, there is an integer $n \in \mathbb{N}$ such that, for all $i, j=1, \ldots, n$,

$$
s_{i} \leq\left(a_{i} a_{j}\right)^{n} s_{j}, s_{j} \leq\left(a_{i} a_{j}\right)^{n} s_{i} .
$$

By Proposition 1.11, $\bigwedge_{i=1}^{n} a_{i}^{n}=1$, and hence we have for $s=\bigwedge_{i=1}^{n} a_{i}^{n} s_{i} \in S$ that $t=\rho_{s}$ in view of the inequalities

$$
s_{j} \leq a_{j}^{n} s=a_{j}^{n}\left(\bigwedge_{i=1}^{n} a_{i}^{n} s_{i}\right)=\bigwedge_{i=1}^{n}\left(a_{i} a_{j}\right)^{n} s_{i}, s \leq a_{j}^{n} s_{j} .
$$

\section{REFERENCES}

[1] D. D. Anderson, Abstract commutative ideal theory without chain condition, Algebra Universalis 6 (1976), 131-145. MR0419310 (54:7332)

[2] D. D. Anderson, Multiplication ideals, multiplication rings, and the ring $R(X)$, Canad. J. Math. 28 (1976), 760-768. MR0424794(54:12752)

[3] P. N. Ánh and M. Siddoway, Divisibility theory of semi-hereditary rings, Proc. Amer. Math. Soc. 138 (2010), 4231-4242. MR2680049

[4] K. E. Aubert, Theory of $x$-ideals, Acta Math. 107 (1962), 1-52. MR0148773 (26:6279)

[5] G. Birkhoff, Lattice Theory, AMS Colloq. Publ. 25, 3rd edition, 1967. MR0227053 (37:2638) 
[6] B. Bosbach, Komplementäre Halbgruppen: Axiomatik und Arithmetik, Fund. Math. 64 (1969), 257-287. MR0260902 (41:5522)

[7] B. Bosbach, Zur Theorie der Teilbarkeitshalbgruppen, Semigroup Forum 3 (1971), 1-30. MR0289384 (44:6575)

[8] B. Bosbach: Concerning bricks, Acta Math. Acad. Sci. Hungar. 38 (1981), 89-104. MR634572 (83m:06021b)

[9] B. Bosbach, Representable divisibility semigroups, Proc. Edinburgh Math. Soc. 34 (1991), 45-64. MR:1093175 (92m:20056)

[10] B. Bosbach, Archimedean Prüfer structures alias filter Archimedean $d$-semigroups, Acta Math. Hungar. 95 (2002), 53-73. MR.1906209 (2003g:06007)

[11] B. Bosbach, Ideal divisibility monoids, Result. Math. 41 (2002), 40-67. MR.1888719 (2003f:06005)

[12] V. Camillo, Distributive modules, J. Algebra 36 (1975), 16-25. MR0573061 (58:28076)

[13] A. H. Clifford, Naturally totally ordered commutative semigroups, Amer. J. Math. 76 (1954), 631-646. MR0062118(15:930b)

[14] R. P. Dilworth, Abstract commutative ideal theory, Pacific J. Math. 12 (1962), 481-498. MR0143781 (26:1333)

[15] M. L. Dubreil-Jacotin, L. Lesieur, and R. Croisot, Leçons sur la Théorie des Treillis, Gauthier-Villars, Paris, 1953. MR0057838 (15:279j)

[16] L. Fuchs, Über die Ideale arithmetischer Ringe, Comment. Math. Helv. 23 (1949), 334-341. MR0032583 (11:310c)

[17] L. Fuchs and L. Salce, Modules over Valuation Domains, Lecture Notes in Pure and Applied Mathematics, 97, Marcel Dekker, New York, 1985. MR786121 (86h:13008)

[18] L. Gillman and M. Henriksen, Rings of continuous functions in which every finitely generated ideal is principal, Trans. Amer. Math. Soc. 82 (1956), 366-391. MR0078980(18:9d)

[19] R. Gilmer, Multiplicative Ideal Theory, Pure and Applied Mathematics, 12, Marcel Dekker, New York, 1972. MR0427289 (55:323)

[20] A. Grothendieck, Éléments de Géométrie Algébrique, I. Le Langage des Schémas, Publ. Math. IHES, 4, Hermann, Paris, 1960. MR0217083 (36:177a)

[21] F. Halter-Koch, Ideal Systems. An Introduction to Multiplicative Ideal Theory, Pure and Applied Mathematics, 211, Marcel Dekker, New York, 1998. MR1828371 (2001m:13005)

[22] M. Henriksen, Some remarks on elementary divisor rings, II, Michigan Math. J. 3 (1955/1956), 159-163. MR0092772(19:1155i)

[23] P. Jaffard, Contribution à la théorie des groupes ordonnés, J. Math. Pures Appl. 32 (1953), 203-280. MR0057869 (15:284d)

[24] P. Jaffard, Les Systèmes d'Idéaux, Dunod, Paris, 1960. MR0114810 (22:5628)

[25] C. U. Jensen, A remark on arithmetical rings, Proc. Amer. Math. Soc. 15 (1964), 951-953. MR0179197 (31:3446)

[26] C. U. Jensen, Arithmetical rings, Acta Math. Acad. Sci. Hungar. 17 (1966), 115-123. MR0190163 (32:7577)

[27] I. Kaplansky, Elementary divisors and modules, Trans. Amer. Math. Soc. 66 (1949), 464-491. MR0031470 (11:155b)

[28] K. Keimel, Darstellung von Halbgruppen und universellen Algebren durch Schnitte in Garben; bireguläre Halbgruppen, Math. Nachr. 45 (1970), 81-96. MR0282907(44:141)

[29] M. D. Larsen, W. J. Lewis and T. S. Shores, Elementary divisor rings and finitely presented modules, Trans. Amer. Math. Soc. 187 (1974), 231-248. MR0335499(49:280)

[30] M. Larsen and P. McCarthy, Multiplicative Theory of Ideals, Academic Press, New York, 1971. MR0414528 (54:2629)

[31] P. Lorenzen, Abstrakte Begründung der multiplikativen Idealtheorie, Math. Z. 45 (1939), 533-553. MR0000604 (1:101c)

[32] E. Matlis, The minimal prime spectrum of a reduced ring, Ill. J. Math. 27 (1983), 353-391. MR698302 (84h:13010)

[33] J. Ohm, Semi-valuations and group of divisibility, Can. J. Math. 21 (1969), 576-591. MR0242819 (39:4146)

[34] R. S. Pierce, Modules over commutative regular rings, Memoirs Amer. Math. Soc. 70 (1967), 112 pp. MR0217056 (36:151)

[35] T. S. Shores, On generalized valuation rings, Michigan Math. J. 21 (1974), 405-409. MR0366892 (51:3138) 
[36] T. S. Shores and R. Wiegand, Rings whose finitely generated modules are direct sums of cyclics, J. Algebra 32 (1974), 152-172. MR0352080(50:4568)

[37] Bo Stenström, Rings of Quotients, Die Grundlehren der mathematischen Wissenschaften in Einzeldarstellungen, 217, Springer, 1975. MR0389953(52:10782)

[38] W. Stephenson, Modules whose lattice of submodules is distributive, Proc. London Math. Soc. 27 (1974), 291-310. MR0338082 (49:2849)

[39] M. H. Stone, Applications of the theory of Boolean rings to general topology, Trans. Amer. Math. Soc. 41 (1937), 375-481. MR.1501905

[40] M. Ward and R. P. Dilworth, Residuated lattices, Trans. Amer. Math. Soc. 45 (1939), 335354. MR1501995

[41] A. Wolf, Sheaf representations of arithmetical algebras, In: Recent Advances in the Representation Theory of Rings and $C^{*}$-Algebras by Continuous Sections, Memoirs Amer. Math. Soc. 148 (1974), 87-93. MR0369223(51:5458)

Rényi Institute of Mathematics, Hungarian Academy of Sciences, 1364 Budapest, Pf. 127 Hungary

E-mail address: anh@renyi.hu

Rényi Institute of Mathematics, Hungarian Academy of Sciences, 1364 Budapest, Pf. 127 Hungary

E-mail address: marki@renyi.hu

Department of Mathematics, University of Exeter, North Park Road, Exeter, EX4 4QF, ENGLAND

E-mail address: p.vamos@exeter.ac.uk 